\title{
Large Deviation Principle for Fractional Brownian Motion with Respect to Capacity
}

\author{
Jiawei $\mathrm{Li}^{1} \cdot$ Zhongmin Qian $^{1}$
}

Received: 10 July 2019 / Accepted: 29 March 2020 / Published online: 28 April 2020

(C) The Author(s) 2020

\begin{abstract}
We show that the fractional Brownian motion (fBM) defined via the Volterra integral representation with Hurst parameter $H \geq \frac{1}{2}$ is a quasi-surely defined Wiener functional on the classical Wiener space, and we establish the large deviation principle (LDP) for such an $\mathrm{fBM}$ with respect to $(p, r)$-capacity on the classical Wiener space in Malliavin's sense.
\end{abstract}

Keywords Capacity · Fractional Brownian motion · Large deviations · Malliavin derivative · Quasi-sure analysis

Mathematics Subject Classifications (2010) 60F10 · 60H07

\section{Introduction}

Quasi-sure analysis, as a powerful tool to study functions on infinite dimensional spaces, was initiated by Malliavin [33-35] using the stochastic calculus of variations and Fukushima [16] by means of Dirichlet forms. The theory can be applied to plentiful aspects in stochastic analysis, such as Markov processes, Gaussian processes, large deviation principles and etc., see e.g. [1, 4, 8, 11, 15, 17-19, 25, 30, 42, 43, 45] and the literature there-in. Malliavin observed that by constructing a regularity theory and a uniform measure on an abstract Wiener space, many interesting Wiener functionals are smooth, and this regularity theory enables us to study Wiener functionals like in the finite dimensional real analysis. An outer measure called $(p, r)$-capacity, denoted by $c_{p, r}$ throughout this paper, was introduced in terms of the Malliavin derivative and a number of papers concerning capacities have been published throughout last decades, see e.g. [15, 25-27, 35-37] and the literature there-in.

Among results related to quasi-sure analysis, the majority considers solutions to Itô's stochastic differential equations, which are merely measurable Wiener functionals. In fact,

Jiawei Li

jiawei.li@maths.ox.ac.uk

Zhongmin Qian

zhongmin.qian@maths.ox.ac.uk

1 Mathematical Institute, University of Oxford, Oxford OX2 6GG, UK 
quasi-sure analysis can be used to handle processes which are measurable functionals on the Wiener space but not solutions to any Itô's SDEs, such as fractional Brownian motions. Fractional Brownian motion (fBM), as an important example of Gaussian processes, has a variety of applications in mathematical finance, hydrodynamics, communication networks and so on, see e.g. Biagini et al. [3], Decreusefond and Üstünel [6], Mandelbrot and Van Ness [38], Mishura [40] and etc. To be more precise, an fBM $\left(B_{t}\right)_{t>0}$ with Hurst parameter $H \in(0,1)$ is a centred self-similar Gaussian process, whose covariance function is given by

$$
\operatorname{Cov}(s, t)=\frac{1}{2}\left(s^{2 H}+t^{2 H}-|t-s|^{2 H}\right) .
$$

By definition, one obtains immediately that when $H=\frac{1}{2}$, this process is a standard Brownian motion. However, when $H$ takes other values, this process differs from Brownian motion as its increments are no longer independent and thus exhibits long memory behaviour. Thanks to the Volterra integral representation introduced by Decreusefond and Üstünel [6], which is

$$
B_{t}=\int_{0}^{t} K(t, s) d W_{s}, \quad \forall t \geq 0,
$$

where $\left(W_{t}\right)_{t \geq 0}$ is a standard Brownian motion and $K$ is some singular kernel, fBMs can be regarded as measurable Wiener functionals. According to Malliavin, fBMs with different Hurst parameters induce a family of capacities living on distinct abstract Wiener spaces. Nonetheless, all these fBMs can be viewed as Wiener functionals on the classical Wiener space due to the integral representation, so that we can study them with one uniform measure - the capacity associated with Brownian motion. In this paper, we will prove that the integral representation of fBM is defined except for a capacity zero set when Hurst parameter $H \geq$ $\frac{1}{2}$, that is, fBMs are quasi-surely defined Wiener functionals on the classical Wiener space.

In order to achieve this goal, we need several results from the rough paths theory. The analysis of rough paths, originated by Lyons (see e.g. [13, 14, 31, 32]), was established to study solutions to stochastic differential equations driven by semi-martingales and other rough signals. It turns out that many techniques developed in the rough paths analysis can be applied in the research of quasi-sure analysis as demonstrated in [4], as well as a series of work by various researchers (see e.g. [21-24, 28, 39] and etc.).

Besides proving that fBMs as Wiener functionals are quasi-surely defined, we establish large deviation principles for these Wiener functionals. Large deviations theory has been a prevalent topic in probability for its significance in statistics and statistical mechanics. It completes the central limit theorem by telling us that tail probabilities decay exponentially fast. In 1970s, the theory of large deviation principles (LDP for short) experienced rapid development due to the remarkable work by Donsker and Varadhan [10], and one may refer to $[7,9,10,44]$ for further details. In finite dimensional case, one crucial result in this theory is the Cramér's theorem, which precisely describes the rate of exponential decay. In infinite dimensional case, the exponential decay of large perturbations of Brownian motion from its mean trajectory is characterised in the Schilder's theorem, and the Freidlin-Wentzell theorem generalises it to the laws of Itô diffusions, see e.g. [7] and [9]. Similar results were proved using rough paths theory by Ledoux, Qian and Zhang in [28], see also [12, 39]. Moreover, the general Cramér's theorem (see e.g. [9]) can be used to study the large deviations of Gaussian measures. Indeed, large deviation principles can be formulated for not only measures, but also capacities. In [45], Yoshida established a version of LDP with respect to capacities on the abstract Wiener space, which implies the LDP for Gaussian measures, while in [17] and [18], Gao and Ren considered the capacity version of Freidlin- 
Wentzell theorem. This line of research was taken a step further to the setting of Gaussian rough paths in [4]. Inspired by the arguments in [4], we prove the LDP for fBMs with respect to $(p, r)$-capacity on the classical Wiener space.

The major difference between this paper and [45] is that we use different capacities. Although the invariance property of capacities has been proved in [2], the capacities associated with different Gaussian measures are however non-comparable. Therefore, instead of using the capacities induced by fBMs, we treat fBMs with different Hurst parameters as a family of Wiener functionals and choose the Brownian motion capacity as a uniform measure.

This paper is organised in the following way. In next section, we present a few definitions and properties of capacities, and then we state the main result, a quasi-sure version of large deviation principle for fractional Brownian motions realised as Wiener functionals on the classical Wiener space. In Section 3, we recall several elementary results such as Wiener chaos decomposition, exponential tightness, contraction principle in the context of quasisure analysis. Then in Section 4, with a quite technical proof, we provide a construction of quasi-surely defined modifications of fBMs, which are considered to be Wiener functionals in our settings. Next, in Section 5, we prove the exponential tightness of the family of finite dimensional approximations of fBMs (modified as in Section 4). In this section, we adopt several ideas from the rough paths theory. Finally, we determine the rate function and complete our proof of the quasi-sure large deviation principles for fBMs in Section 6. The key step is to obtain the finite dimensional quasi-sure large deviation principles, which may be accomplished by explicit computations.

\section{Preliminaries and the Main Result}

In this section, we will introduce basic definitions in Malliavin calculus and state the main result.

\subsection{Malliavin Differentiation and Capacities}

We mainly follow the notations used in Ikeda and Watanabe [20], and Nualart [41]. Although our presentation applies to multi-dimensional case as well, we only consider the one dimensional Wiener space here for simplicity. Let $\boldsymbol{W}$ be the space of all real-valued continuous paths over time interval $[0,1]$ starting from the origin, equipped with the uniform norm $\|\cdot\|$ given by $\|\omega\|=\sup _{t \in[0,1]}|\omega(t)|, \forall \omega \in \boldsymbol{W}$. The Borel $\sigma$-algebra is denoted by $\mathscr{B}(\boldsymbol{W})$. We call the functions which send each $\omega \in \boldsymbol{W}$ to its coordinates $\omega(t)$ (where $t \geq 0$ ) the coordinate mapping processes, and are denoted by $\omega(t)$ or $\omega_{t}$. The Wiener measure $\mathbb{P}$ is the distribution of standard Brownian motion, the unique probability on $(\boldsymbol{W}, \mathscr{B}(\boldsymbol{W}))$ such that $\{\omega(t): t \geq 0\}$ is a standard Brownian motion. Let $\mathscr{F}$ be the completion of $\mathscr{B}(\boldsymbol{W})$ under $\mathbb{P}$. The Wiener functionals, by convention in the literature, are $\mathscr{F}$-measurable functions on $\boldsymbol{W}$.

Let $\mathscr{H}$ denote the Cameron-Martin space, which is a Hilbert space containing all absolutely continuous functions $h$ on $[0,1]$ such that $h(0)=0$ and its generalised derivative $\dot{h}$ is square integrable. The inner product on $\mathscr{H}$ is given by

$$
\langle h, g\rangle_{\mathscr{H}}=\int_{0}^{1} \dot{h}(s) \dot{g}(s) d s, \quad \forall h, g \in \mathscr{H}
$$


This space may be embedded into $\boldsymbol{W}$ via a continuous and dense embedding. Denote the topological dual of $\boldsymbol{W}$ by $\boldsymbol{W}^{*}$. Then we have continuous dense embeddings $\boldsymbol{W}^{*} \hookrightarrow \mathscr{H} \hookrightarrow$ $\boldsymbol{W}$. The triple $(\boldsymbol{W}, \mathscr{H}, \mathbb{P})$ is called the classical Wiener space.

There is a natural linear isometry from $\mathscr{H}$ to $L^{2}(\boldsymbol{W})$ sending each element $h \in \mathscr{H}$ to a random variable $[h]$ such that $[h](\omega)=\int_{0}^{1} \dot{h}(s) d \omega(s)$ for all $\omega \in \boldsymbol{W}$, which is defined as an Itô's integral with respect to Brownian motion. Then $\mathbb{E}[[h][g]]=\langle h, g\rangle_{\mathscr{H}}$. The family $\{[h]: h \in \mathscr{H}\}$ is called the Gaussian isometry process in [41] (see Section 1.1.1, Chapter $1,[41])$.

A random variable $F$ on $\boldsymbol{W}$ is smooth if it is of the form $F=f\left(\left[h_{1}\right], \cdots,\left[h_{n}\right]\right)$ for some $h_{1}, \cdots h_{n} \in \mathscr{H}$ and $f \in C_{p}^{\infty}\left(\mathbb{R}^{n}\right)$, a smooth function such that $f$ and all of its partial derivatives have polynomial growth. The collection of all smooth random variables is denoted by $\mathcal{S}$. The Malliavin derivative $D F$ of $F$ is defined to be an $\mathscr{H}$-valued random variable, given by

$$
D F=\sum_{i=1}^{n} \partial_{i} f\left(\left[h_{1}\right], \cdots,\left[h_{n}\right]\right) h_{i},
$$

where $\partial_{i} f$ denotes the partial derivative of $f$ in the $i$-th component. The higher order derivatives, $D^{l} F, l \geq 1$, are defined inductively. The Sobolev norm $\|\cdot\|_{\mathbb{D}_{r}^{p}}$ of a smooth random variable $F$ is defined as

$$
\|F\|_{\mathbb{D}_{r}^{p}}=\left(\mathbb{E}\left[|F|^{p}\right]+\sum_{l=1}^{r} \mathbb{E}\left[\left|\left\|D^{l} F\right\|_{\mathscr{H} \otimes l}\right|^{p}\right]\right)^{1 / p},
$$

where $r=0,1,2, \cdots$ and $1 \leq p<\infty$. The completion of $\mathcal{S}$ with respect to this norm is denoted by $\mathbb{D}_{r}^{p}$.

The concept of capacities on the classical Wiener space plays a central role in what follows. For given $p \geq 1$ and $r=0,1,2, \cdots$, the capacity $c_{p, r}$ is a sub-additive set function on the classical Wiener space, which can be defined in two steps.

First for every open $O \subset \boldsymbol{W}$ (see e.g. [35]), set

$$
c_{p, r}(O)=\inf \left\{\|\varphi\|_{\mathbb{D}_{r}^{p}}: \varphi \in \mathbb{D}_{r}^{p}, \varphi \geq 1 \text { a.e. on } O, \varphi \geq 0 \text { a.e. on } \boldsymbol{W}\right\} .
$$

Next for an arbitrary subset $A$ of $\boldsymbol{W}$,

$$
c_{p, r}(A)=\inf \left\{c_{p, r}(O): A \subset O, O \text { is open }\right\} .
$$

A property $\pi=\pi(\omega)$ (whose description depends on $\omega \in \boldsymbol{W}$ ) holds $(p, r)$-quasi-surely (or simply ( $p, r)$-q.s.), if the set on which $\pi$ is not satisfied has $(p, r)$-capacity zero. A property $\pi$ is said to hold quasi-surely (q.s.) if it holds $(p, r)$-quasi-surely for all $r=0,1,2, \cdots$ and $1<p<\infty$. A set is said to be slim if it has zero $(p, r)$-capacity for all $r=0,1,2, \cdots$ and $1<p<\infty$.

Let us recall a few elementary properties of capacities $c_{p, r}$ (see e.g. Section 1.2, Chapter $\mathrm{IV},[35])$. By definition every capacity is an outer measure, so that $c_{p, r}(A) \leq c_{p, r}(B)$ for $A \subset B \subset \boldsymbol{W}$, and $c_{p, r}$ is sub-additive in that

$$
c_{p, r}\left(\bigcup_{n=1}^{\infty} A_{n}\right) \leq \sum_{n=1}^{\infty} c_{p, r}\left(A_{n}\right)
$$

for any $A_{n} \subset \boldsymbol{W}, n=1,2, \cdots$.

The family of capacities $c_{p, r}$ are comparable in the sense that for $\forall A \subset \boldsymbol{W}, 1<p<$ $q<\infty$, and $r<s$, we have $c_{p, r}(A) \leq c_{q, r}(A)$ and $c_{p, r}(A) \leq c_{p, s}(A)$. In particular, by definition, $(\mathbb{P}(A))^{\frac{1}{p}} \leq c_{p, r}(A)$ for any $A \in \mathscr{F}$. 
The first Borel-Cantelli lemma can be proved in the context of capacities, and it says that if a sequence of subsets $\left\{A_{n}\right\}_{n=1}^{\infty}$ of $\boldsymbol{W}$ satisfies $\sum_{n=1}^{\infty} c_{p, r}\left(A_{n}\right)<\infty$, then $c_{p, r}\left(\lim \sup _{n \rightarrow \infty} A_{n}\right)=0$. One may refer to [35] for a proof.

Another important tool used in this paper is the capacity version of Chebyshev's inequality, which is

$$
c_{p, r}(\varphi>\lambda) \leq \frac{\|\varphi\|_{\mathbb{D}_{r}^{p}}}{\lambda},
$$

for every lower semi-continuous $\varphi \in \mathbb{D}_{r}^{p}$ and $\lambda>0$.

\subsection{The Main Result}

We are now ready to introduce the definition of large deviation principle (LDP) with respect to capacities as in [4] and [45]. Then we define fractional Brownian motions and its integral representation according to [6] (see also Chapter 5 in [41]) and state the main result at the end of this section.

Definition 1 Let $r \in \mathbb{N}$ and $p>1$, and $\left\{X^{\varepsilon}: \varepsilon>0\right\}$ be a family of $(p, r)$-quasi-surely defined mappings from $\boldsymbol{W}$ to a Polish space $(Y, d)$. We say that the family $\left\{X^{\varepsilon}: \varepsilon>0\right\}$ satisfies the $c_{p, r}$-large deviation principle $\left(c_{p, r}\right.$-LDP) with a good rate function $I: Y \rightarrow$ $[0, \infty]$ if

(1) $I$ is lower semi-continuous and for every $\alpha>0$, the level set

$$
\Psi_{I}(\alpha)=\{y \in Y: I(y) \leq \alpha\}
$$

is compact in $Y$; and

(2) for every closed $F \subset Y$,

$$
\limsup _{\varepsilon \rightarrow 0} \varepsilon^{2} \log c_{p, r}\left\{\omega \in \boldsymbol{W}: X^{\varepsilon}(\omega) \in F\right\} \leq-\frac{1}{p} \inf _{y \in F} I(y),
$$

for every open $G \subset Y$,

$$
\limsup _{\varepsilon \rightarrow 0} \varepsilon^{2} \log c_{p, r}\left\{\omega \in \boldsymbol{W}: X^{\varepsilon}(\omega) \in G\right\} \geq-\frac{1}{p} \inf _{y \in G} I(y) .
$$

Recall that a fractional Brownian motion (fBM) $\left(B_{t}\right)_{t \geq 0}$ with Hurst parameter $H \in(0,1)$ is a self-similar Gaussian process with stationary increments whose covariance function is given by

$$
\operatorname{Cov}(s, t)=\mathbb{E}\left[B_{s} B_{t}\right]=\frac{1}{2}\left(t^{2 H}+s^{2 H}-|t-s|^{2 H}\right) .
$$

FBMs can be realised as Wiener functionals in the way that

$$
B_{t}(\omega)=\int_{0}^{t} K(t, s) d \omega(s),
$$

where $\{\omega(s): s \geq 0\}$ is the coordinate mapping process on $\boldsymbol{W}$ (hence a Brownian motion). $B_{t}$ is defined almost surely - the integral on the right-hand side is understood in the Itô's sense. $K$ is a singular kernel, and when $H>\frac{1}{2}$, it is given by

$$
K(t, s)=c_{H} s^{\frac{1}{2}-H} \int_{s}^{t}(u-s)^{H-\frac{3}{2}} u^{H-\frac{1}{2}} d u, \quad s<t,
$$

where $c_{H}$ is some constant depending only on $H$. It is straightforward by the Kolmogorov continuity theorem that the process defined by Eq. 1 has a modification which is $\alpha$-Hölder 
continuous with $\alpha<H$. We abuse our notation by using $\left(B_{t}\right)_{t \geq 0}$ to denote such a modification, and in the sequel, when we mention $\mathrm{fBM}$, we always refer to this modified version.

To state the quasi-sure large deviation principles for fBMs, we need to identify the corresponding rate functions, which must be the same rate functions as in the context of probabilities. Notice that (1) defines a mapping $B: \boldsymbol{W} \rightarrow \boldsymbol{W}$ taking almost all Brownian motion paths to the paths of fBM with Hurst parameter $H$. Let $\mathbb{Q}=\mathbb{P} \circ B^{-1}$ be the pushforward of the Wiener measure, which is the distribution of this version of $\mathrm{fBM}$, a Gaussian measure on $(\boldsymbol{W}, \mathscr{B}(\boldsymbol{W}))$. Similar to the case of Brownian motion, there is a canonical way to associate this Gaussian measure and $\boldsymbol{W}$ with a separable Hilbert space $\hat{\mathscr{H}}$, which can be continuously and densely embedded into $\boldsymbol{W}$ (see e.g. [5]). In [6], the Cameron-Martin space $\hat{\mathscr{H}}$ corresponding to the fractional Brownian motion with Hurst parameter $H$ was identified, and is the space consisting of all elements of the form $h(t)=\int_{0}^{t} K(t, s) \dot{h}(s) d s$, where $K$ is as in Eq. 2, and $\dot{h} \in L^{2}([0,1])$. The inner product on $\hat{\mathscr{H}}$ is defined as

$$
\left\langle h_{1}, h_{2}\right\rangle_{\hat{\mathscr{H}}}=\int_{0}^{1} \dot{h}_{1}(s) \dot{h}_{2}(s) d s,
$$

where $h_{i}(t)=\int_{0}^{t} K(t, s) \dot{h}_{i}(s) d s, i=1,2$. Then

$$
\int_{W} e^{i l(\omega)} \mathbb{Q}(d \omega)=e^{-\frac{\|l\|^{2}}{2} \hat{\mathscr{e}}}, \quad \forall l \in W^{*} .
$$

Let $\left\{\mathbb{Q}_{\varepsilon}\right\}$ be the family of scaled measures, the laws of $\{\varepsilon \omega\}$ under $\mathbb{Q}$. By definition

$$
\mathbb{Q}_{\varepsilon}(A)=\mathbb{Q}\{\omega \in \boldsymbol{W}: \varepsilon \omega \in A\}=\mathbb{P}\{\omega \in \boldsymbol{W}: \varepsilon B(\omega) \in A\}
$$

for each $A \in \mathscr{B}(\boldsymbol{W})$. According to Theorem 3.4.12 on page 88 in [9], $\left\{\mathbb{Q}_{\varepsilon}\right\}$ satisfies the LDP with the good rate function $I$ given by

$$
I(\omega)= \begin{cases}\frac{\|\omega\|^{2} \hat{\mathscr{H}}}{2}, & \omega \in \hat{\mathscr{H}}, \\ \infty, & \text { otherwise. }\end{cases}
$$

Now we are in a position to state the main result of the present paper. Let $\left(B_{t}\right)_{t \geq 0}$ be the continuous modification of fBM given by Eq. 1 with Hurst parameter $H$.

Theorem 2 Let $r \in \mathbb{N}, p>1$ and $\frac{1}{2} \leq H<1$. Then we have the following conclusions.

(1) There exists a modification of $B_{t}$ (for $t \geq 0$ ) defined $(p, r)$-quasi-surely.

(2) Let $X_{t}^{\varepsilon}(\omega)=B_{t}(\varepsilon \omega)$ for all $\omega$ expect for a $c_{p, r}$-zero subset (for all $t \geq 0$, $\varepsilon>0$ ). Then $\left\{X^{\varepsilon}: \varepsilon>0\right\}$ (which are scaled fBMs with Hurst parameter $H \in\left[\frac{1}{2}, 1\right.$ )) satisfies the $c_{p, r}$-LDP with the good rate function

$$
I(\omega)= \begin{cases}\frac{\|\omega\|^{2} \hat{\mathscr{H}}}{2}, & \omega \in \hat{\mathscr{H}}, \\ \infty, & \text { otherwise. }\end{cases}
$$

The remaining of this paper is devoted to the proof of the above result, Theorem 2 . The first part of Theorem 2 follows from Theorem 8 in Section 4 directly. The proof of the second part will be presented in Sections 5 and 6.

Our strategy of the proof is the following. For each fixed $t \in[0,1]$ and $m \in \mathbb{N}$, we consider $B_{t}^{(m)}$, a finite linear combination of elements in the classical Wiener space, and show that the sequence $\left(B_{t}^{(m)}\right)_{m \in \mathbb{N}}$ converges quasi-surely (with respect to the Brownian 
motion capacity). The main difficulty here is that the kernel $K(t, s)$ is singular in $s$, so it is very difficult to control its increments in $s$ and estimate the integral of $K$ over small time intervals near time $s=0$. However, we notice that $K$ as a function of $t$, behaves more regularly. Therefore, we control the difference $K(t, s+\alpha)-K(t, s)$ by the difference of $K$ when $t$ varies. Then we may obtain the desired mapping $X$, a quasi-surely defined modification of fBM on the Wiener space by approximations with linear interpolations. Define

$$
X^{(n)}(\omega)(t):=B_{\frac{k-1}{2^{n}}}(\omega)+2^{n}\left(t-\frac{k-1}{2^{n}}\right)\left(B_{\frac{k}{2^{n}}}(\omega)-B_{\frac{k-1}{2^{n}}}(\omega)\right), \quad \forall \frac{k-1}{2^{n}} \leq t \leq \frac{k}{2^{n}} .
$$

For each $n, X^{(n)}$ is quasi-surely defined, then we show that this sequence $\left(X^{(n)}\right)_{n \in \mathbb{N}}$ converges to some mapping $X$ quasi-surely. Since any countable union of capacity zero sets still has zero capacity, we conclude that the limit $X$ is quasi-surely defined, and we shall also see that this convergence is exponentially fast in the proof. Now as the large deviation principle may be established for $X^{(n)}$ 's, using exponentially good approximations result from the LDP theory, we deduce the $c_{p, r}$-LDP for the limit mapping $X$.

\section{Some Technical Facts}

In this section we collect a few technical facts which will be used to prove the quasi-sure version of large deviation principles for fBMs.

\subsection{Wiener Chaos Decomposition}

The $n$-th Wiener chaos $\mathcal{H}_{n}, n=0,1,2, \cdots$, is the closed subspace of $L^{2}(\boldsymbol{W})$ generated by all random variables of the form $\Phi_{n}=\prod_{i=1}^{m} H_{k_{i}}\left(\left[h_{i}\right]\right)$, where $k_{1}+\cdots+k_{m}=n$ and $h_{i} \in \mathscr{H}$ with $\left\|h_{i}\right\|_{\mathscr{H}}=1$. Here, $H_{n}(x)$ denotes the $n$-th Hermite polynomial. $\mathcal{H}_{n}$ and $\mathcal{H}_{m}$ are orthogonal subspaces of $L^{2}(\boldsymbol{W})$ when $m \neq n$. One important result is the Wiener chaos decomposition, which states that

$$
L^{2}(\boldsymbol{W}, \mathscr{G}, \mathbb{P})=\oplus_{n=0}^{\infty} \mathcal{H}_{n},
$$

where $\mathscr{G}$ is the $\sigma$-algebra generated by random variables $\{[h]: h \in \mathscr{H}\}$ (see e.g. Theorem 1.1.1, Section 1.1 in [41] and its proof). The projection from $L^{2}(\boldsymbol{W})$ to $\mathcal{H}_{n}$ is denoted by $J_{n}$. Let $\mathcal{P}_{n}^{0}$ denote the space of polynomial random variables of the form

$$
F=p\left(\left[h_{1}\right], \cdots\left[h_{k}\right]\right), \quad \forall h_{1}, \cdots h_{k} \in \mathscr{H},
$$

where $p$ is a polynomial with degree less than or equal to $n$, and let $\mathcal{P}_{n}$ be the closure of $\mathcal{P}_{n}^{0}$ in $L^{2}(\boldsymbol{W})$. Then it follows that for every integer $n, \mathcal{P}_{n}=\oplus_{m=0}^{n} \mathcal{H}_{m}$. If $F \in \mathbb{D}_{r}^{p}$ for some $p \geq 2$, then for all $l \leq r$,

$$
\|\| D^{l} F\left\|_{\mathscr{H} \otimes l}\right\|_{2}^{2}=\sum_{n=l}^{\infty} n(n-1) \cdots(n-l+1)\left\|J_{n} F\right\|_{2}^{2} .
$$

For a proof of this relation, one may refer to Section 1.2 in [41]. 
To establish next proposition, we need to apply the hypercontractivity property of Ornstein-Uhlenbeck semigroup. The Ornstein-Uhlenbeck semigroup $\left(T_{t}\right)_{t \geq 0}$ is a semigroup of contractions on $L^{2}(\boldsymbol{W})$ defined by

$$
T_{t} F=\sum_{n=0}^{\infty} e^{-n t} J_{n} F
$$

This definition can be extended to $L^{p}(\boldsymbol{W})$ and for every $p>1,\left(T_{t}\right)_{t \geq 0}$ is defined to be a semigroup of contractions on $L^{p}(\boldsymbol{W})$, where for each $t \geq 0$,

$$
T_{t} F(\omega)=\int_{W} F\left(e^{-t} \omega+\sqrt{1-e^{-2 t}} x\right) \mathbb{P}(d x) .
$$

This semigroup enjoys the hypercontractivity property, which is $\left\|T_{t} F\right\|_{q(t)} \leq\|F\|_{p}$ for all $F \in L^{p}(\boldsymbol{W})$, where $p>1, t>0$ and $q(t)=e^{2 t}(p-1)+1>p$. For a proof, see e.g. Theorem 1.4.1, Section 1.4, [41].

Proposition 3 Let $F \in \mathcal{P}_{n}$. Then

$$
\|F\|_{q} \leq(n+1)(q-1)^{\frac{n}{2}}\|F\|_{2}
$$

and

$$
\|\| D^{l} F\left\|_{\mathscr{H} \otimes l}\right\|_{2} \leq n^{\frac{l}{2}}\|F\|_{2}
$$

for any $q>2$ and $l \leq n$.

Proof The first inequality (6) results from hypercontractivity. Take $F \in \mathcal{H}_{n}$. Then $T_{t} F=$ $e^{-n t} F$. Set $p=2$, and $q=q(t)=1+e^{2 t}$, so $t=\frac{1}{2} \log (q-1)$, and hence by hypercontractivity,

$$
\left\|T_{t} F\right\|_{q}=\left\|e^{-\frac{n}{2} \log (q-1)} F\right\|_{q}=(q-1)^{-\frac{n}{2}}\|F\|_{q} \leq\|F\|_{2},
$$

which implies that

$$
\|F\|_{q} \leq(q-1)^{\frac{n}{2}}\|F\|_{2} .
$$

Now let $F=\sum_{m=0}^{n} J_{m} F \in \mathcal{P}_{n}$, then

$$
\|F\|_{q} \leq \sum_{m=0}^{n}\left\|J_{m} F\right\|_{q} \leq \sum_{m=0}^{n}(q-1)^{\frac{m}{2}}\left\|J_{m} F\right\|_{2} \leq(n+1)(q-1)^{\frac{n}{2}}\|F\|_{2} .
$$

To prove (7), we apply (5) to $F \in \mathcal{P}_{n}$, where $F \in \mathbb{D}_{l}^{2}$ for $l \leq n$. Notice that $J_{m} F$ 's vanish when $m>n$, and thus $\|F\|_{2}^{2}=\sum_{m=0}^{n}\left\|J_{m} F\right\|_{2}^{2}$, so that

$$
\|\| D^{l} F\left\|_{\mathscr{H} \otimes l}\right\|_{2}^{2}=\sum_{m=l}^{n} m(m-1) \cdots(m-l+1)\left\|J_{m} F\right\|_{2}^{2} \leq n^{l} \sum_{m=l}^{n}\left\|J_{m} F\right\|_{2}^{2} \leq n^{l}\|F\|_{2}^{2} .
$$

\subsection{Exponential Tightness}

Most conclusions in the theory of large deviations (see [7,9] for details) are still valid in the context of capacities. Let us state some of them which will be used in this paper, their proofs are routine and will be omitted. 
Proposition 4 (Varadhan's Contraction Principle) Let $\left\{X^{\varepsilon}: \varepsilon>0\right\}$ be a family of $(p, r)$ quasi-surely defined maps from $\boldsymbol{W}$ to a Polish space $\left(Y_{1}, d_{1}\right)$ satisfying the $c_{p, r}-L D P$ with the good rate function $I$. Let $F$ be a continuous map from $\left(Y_{1}, d_{1}\right)$ to another Polish space $\left(Y_{2}, d_{2}\right)$. Then the family $\left\{F \circ X^{\varepsilon}: \varepsilon>0\right\}$ of $(p, r)$-quasi-surely defined maps satisfies the $c_{p, r}-L D P$ with the good rate function

$$
J(z)=\inf _{y: F(y)=z} I(y),
$$

where $\inf \emptyset=\infty$.

To deal with fBMs, which are merely measurable Wiener functionals, the concept of exponential tightness is a useful technique in proving large deviation principles. The natural modification of this notion can be formulated as the following.

Definition 5 For $m=1,2, \cdots$, let $\left\{X^{\varepsilon,(m)}: \varepsilon>0\right\}$ and $\left\{X^{\varepsilon}: \varepsilon>0\right\}$ be families of $(p, r)$ quasi-surely defined mappings from $\boldsymbol{W}$ to some Polish space $(Y, d)$. Then $\left\{X^{\varepsilon,(m)}: \varepsilon>0\right\}$ is said to be a family of exponentially good approximations of $\left\{X^{\varepsilon}: \varepsilon>0\right\}$ under $(p, r)$ capacity if for all $\lambda>0$,

$$
\lim _{m \rightarrow \infty} \limsup _{\varepsilon \rightarrow 0} \varepsilon^{2} \log c_{p, r}\left\{\omega: d\left(X^{\varepsilon,(m)}(\omega), X^{\varepsilon}(\omega)\right)>\lambda\right\}=-\infty .
$$

The following version of the contraction principle will be useful in our proof.

Proposition 6 Suppose that for each $m=1,2, \cdots$, the family $\left\{X^{\varepsilon,(m)}: \varepsilon>0\right\}$, consisting of $(p, r)$-quasi-surely defined mappings from $\boldsymbol{W}$ to $(Y, d)$, satisfies $c_{p, r}$-LDP with the good rate function $I_{m}$, and $\left\{X^{\varepsilon,(m)}: \varepsilon>0\right\}$ are exponentially good approximations of $(p, r)$ quasi-surely defined mappings $\left\{X^{\varepsilon}: \varepsilon>0\right\}$. Define the function

$$
J(y)=\sup _{\lambda>0} \liminf _{m \rightarrow \infty} \inf _{z \in B(y, \lambda)} J_{m}(z), \quad \forall y \in Y,
$$

where $B(y, \lambda)$ denotes the open ball in $(Y, d)$ with centre $y$ and radius $\lambda$. If $J$ is a good rate function and for every closed $C \subset Y$,

$$
\inf _{y \in C} J(y) \leq \limsup _{m \rightarrow \infty} \inf _{y \in C} J_{m}(y),
$$

then the family $\left\{X^{\varepsilon}: \varepsilon>0\right\}$ satisfies $c_{p, r}-L D P$ with the good rate function $J$.

\section{FBMs as Wiener Functionals}

Let $B=\left(B_{t}\right)_{t \geq 0}$ the continuous version of fBM with Hurst parameter $H$ defined via (1). According to the transfer principle in [41] (see Proposition 5.2.1, Section 5.2, [41]), $B_{t} \in$ $\mathbb{D}_{r}^{p}$, and its Malliavin derivative may be computed explicitly as in the following lemma (see also [29]).

Lemma 7 Let $H \in(0,1), r \in \mathbb{N}$ and $p \in(1, \infty)$. Then for every $t>0, B_{t} \in \mathbb{D}_{r}^{p}$ and its first order Malliavin derivative is given by

$$
D B_{t}(s)=\int_{0}^{s \wedge t} K(t, u) d u .
$$

The higher order derivatives of $B_{t}$ vanish. 
For every $t>0$, and $m=1,2, \cdots$, define

$$
B_{t}^{(m)}(\omega):=\sum_{i=0}^{2^{m}-1} \frac{2^{m}}{t} \int_{\frac{i}{2^{m}} t}^{\frac{i+1}{2^{m}} t} K(t, r) d r\left(\omega_{\frac{i+1}{2^{m}} t}-\omega_{\frac{i}{2^{m}} t}\right),
$$

where $B_{0}^{(m)}=0$. Obviously $B_{t}^{(m)} \in \mathbb{D}_{r}^{p}$ and

$$
D B_{t}^{(m)}(s)=u_{t}^{(m)}=\sum_{i=0}^{2^{m}-1} \frac{2^{m}}{t} \int_{\frac{i}{2^{m}} t}^{\frac{i+1}{2^{m} t}} K(t, r) d r \mathbb{1}\left(\frac{i}{2^{m}} t, \frac{i+1}{2^{m}} t\right]^{(s)} .
$$

Its higher order Malliavin derivatives vanish identically.

The first part of Theorem 2 is a consequence of the following result.

Theorem 8 Let $H \in\left[\frac{1}{2}, 1\right)$. For all $r \in \mathbb{N}, 1<p<\infty$ and $t \in[0,1],\left(B_{t}^{(m)}\right)_{m \in \mathbb{N}}$ converges $(p, r)$-quasi-surely to some limit, denoted by $B_{t}$ too, which is also the limit of $\left(B_{t}^{(m)}\right)_{m \in \mathbb{N}}$ in $\mathbb{D}_{r}^{p}$.

Proof The proof is quite technical and will be divided into several steps. When $H=\frac{1}{2}$, an fBM is a standard Brownian motion, and hence the result follows immediately. We only need to consider the case when $H>\frac{1}{2}$. Let us begin with a simple fact that

$$
\left\{\omega:\left(B_{t}^{(m)}(\omega)\right)_{m \in \mathbb{N}} \text { is not Cauchy }\right\} \subset \limsup _{m \rightarrow \infty}\left\{\omega:\left|B_{t}^{(m+1)}(\omega)-B_{t}^{(m)}(\omega)\right|>\frac{1}{2^{m \delta}}\right\}
$$

for some $\delta>0$. Therefore, by the first Borel-Cantelli lemma for capacities, we only need to show that

$$
\sum_{m=1}^{\infty} c_{p, r}\left(\left|B_{t}^{(m+1)}-B_{t}^{(m)}\right|>\frac{1}{2^{m \delta}}\right)<\infty
$$

for all $p \in(1, \infty)$ and $r \in \mathbb{N}$. Since $c_{p, r}$ is increasing in $p$ and $r$, it suffices to prove that the above infinite sum is finite for $p>2$ and all $r \in \mathbb{N}$. Therefore, we shall assume that $p>2$ in the sequel.

Step 1. In this step, we convert our problem from estimating the capacities to estimating the $L^{2}$-norm of Gaussian random variables. By Chebyshev's inequality, we have

$$
\begin{aligned}
c_{p, r}\left(\left|B_{t}^{(m+1)}-B_{t}^{(m)}\right|>\lambda\right) & =c_{p, r}\left(\left|B_{t}^{(m+1)}-B_{t}^{(m)}\right|^{2}>\lambda^{2}\right) \\
& \leq \lambda^{-2}\left\|\left(B_{t}^{(m+1)}-B_{t}^{(m)}\right)^{2}\right\|_{\mathbb{D}_{r}^{p}}
\end{aligned}
$$

for any $\lambda>0$. Since $\left(B_{t}^{(m+1)}-B_{t}^{(m)}\right)^{2}$ is a polynomial functional of degree 2 , and

$$
D\left(B_{t}^{(m+1)}-B_{t}^{(m)}\right)=u_{t}^{(m+1)}-u_{t}^{(m)},
$$

where $u_{t}^{(m)}$ is defined as in Eq. 12, so for all $l \geq 3$,

$$
D^{l}\left(\left(B_{t}^{(m+1)}-B_{t}^{(m)}\right)^{2}\right)=0 .
$$


Therefore, by Eq. 3,

$$
\begin{aligned}
\|\| D^{l}\left(\left(B_{t}^{(m+1)}-B_{t}^{(m)}\right)^{2}\right)\left\|_{\mathscr{H}^{\otimes l}}\right\|_{p} & \leq 3(p-1)\|\| D^{l}\left(\left(B_{t}^{(m+1)}-B_{t}^{(m)}\right)^{2}\right)\left\|_{\mathscr{H}^{\otimes l}}\right\|_{2} \\
& \leq 3(p-1) 2^{\frac{l}{2}}\left\|\left(B_{t}^{(m+1)}-B_{t}^{(m)}\right)^{2}\right\|_{2} \\
& =3(p-1) 2^{\frac{l}{2}}\left\|B_{t}^{(m+1)}-B_{t}^{(m)}\right\|_{4}^{2} \\
& \leq 3(p-1) 2^{\frac{l}{2}}\left(2 \sqrt{3}\left\|B_{t}^{(m+1)}-B_{t}^{(m)}\right\|_{2}\right)^{2} \\
& =36(p-1) 2^{\frac{l}{2}}\left\|B_{t}^{(m+1)}-B_{t}^{(m)}\right\|_{2}^{2}
\end{aligned}
$$

for all $p>2$ and $0 \leq l \leq r$, and therefore

$$
\begin{aligned}
\left\|\left(B_{t}^{(m+1)}-B_{t}^{(m)}\right)^{2}\right\|_{\mathbb{D}_{r}^{p}} & \leq \sum_{l=0}^{r}\|\| D^{l}\left(B_{t}^{(m+1)}-B_{t}^{(m)}\right)\left\|_{\mathscr{H}^{\otimes l} l}\right\|_{p} \\
& \leq C_{r, p}\left\|B_{t}^{(m+1)}-B_{t}^{(m)}\right\|_{2}^{2}
\end{aligned}
$$

where

$$
C_{r, p}=36(r+1)(p-1) 2^{\frac{r}{2}}
$$

depends only on $r$ and $p$. The $L^{2}$-norm on the right-hand side of Eq. 14 can be handled as the following. By definition (11),

$$
\begin{aligned}
B_{t}^{(m+1)}(\omega)-B_{t}^{(m)}(\omega)= & \sum_{i=0}^{2^{m+1}-1} \frac{2^{m+1}}{t} \int_{\frac{i}{2^{m+1}} t}^{\frac{i+1}{2^{m+1}} t} K(t, r) d r\left(\omega_{\frac{i+1}{2^{m+1}} t}-\omega_{\frac{i}{2^{m+1}} t}\right) \\
& -\sum_{i=0}^{2^{m}-1} \frac{2^{m}}{t} \int_{\frac{i}{2^{m}} t}^{\frac{i+1}{2^{m}} t} K(t, r) d r\left(\omega_{\frac{i+1}{2^{m}} t}-\omega_{\frac{i}{2^{m}} t}\right) .
\end{aligned}
$$

The integral term in $B_{t}^{(m)}(\omega)$ may be split into two parts, i.e. for each $i \in\left\{0,1, \cdots, 2^{m}-1\right\}$,

$$
\begin{aligned}
& \frac{2^{m}}{t} \int_{\frac{i}{2^{m}} t}^{\frac{i+1}{2^{m}} t} K(t, r) d r\left(\omega_{\frac{i+1}{2^{m}} t}-\omega_{\frac{i}{2^{m}} t}\right) \\
= & \frac{2^{m}}{t}\left[\int_{\frac{2 i+1}{2^{m+1}} t}^{\frac{2 i+2}{2^{m+1}} t} K(t, r) d r\left(\omega_{\frac{2 i+2}{2^{m+1}} t}-\omega_{\frac{2 i}{2^{m+1}} t}\right)+\int_{\frac{2 i}{2^{m+1}} t}^{\frac{2 i+1}{2^{m+1}} t} K(t, r) d r\left(\omega_{\frac{2 i+2}{2^{m+1}} t}-\omega_{\frac{2 i}{2^{m+1}} t}\right)\right],
\end{aligned}
$$

and the contribution from the interval $\left(\frac{i}{2^{m}} t, \frac{i+1}{2^{m}} t\right]$ in $B_{t}^{(m+1)}(\omega)$ is

$$
\frac{2^{m+1}}{t}\left[\int_{\frac{2 i+1}{2^{m+1}} t}^{\frac{2 i+2}{2^{m+1}} t} K(t, r) d r\left(\omega_{\frac{2 i+2}{2^{m+1}} t}-\omega_{\frac{2 i+1}{2^{m+1}} t}\right)+\int_{\frac{2 i}{2^{m+1}} t}^{\frac{2 i+1}{2^{m+1}} t} K(t, r) d r\left(\omega_{\frac{2 i+1}{2^{m+1}} t}-\omega_{\frac{2 i}{2^{m+1}} t}\right)\right] .
$$


Therefore, we deduce that

$$
\begin{aligned}
& B_{t}^{(m+1)}(\omega)-B_{t}^{(m)}(\omega) \\
= & \frac{2^{m}}{t} \sum_{i=0}^{2^{m}-1}\left[\int_{\frac{2 i+1}{2^{m+1}} t}^{\frac{2 i+2}{2^{m+1}} t} K(t, r) d r\left(\omega_{\frac{2 i+2}{2^{m+1}} t}-2 \omega_{\frac{2 i+1}{2^{m+1}} t}+\omega_{\frac{2 i}{2^{m+1}} t}\right)\right. \\
& \left.+\int_{\frac{2 i}{2^{m+1}} t}^{\frac{2 i+1}{2^{m+1}} t} K(t, r) d r\left(2 \omega_{\frac{2 i+1}{2^{m+1}} t}-\omega_{\frac{2 i}{2^{m+1}} t}-\omega_{\frac{2 i+2}{2^{m+1}} t}\right)\right] \\
= & \frac{2^{m}}{t} \sum_{i=0}^{2^{m}-1}\left(\int_{\frac{2 i+1}{2^{m+1}} t}^{\frac{2 i+2}{2^{m+1}} t} K(t, r) d r-\int_{\frac{2 i}{2^{m+1}} t}^{\frac{2 i+1}{2^{m+1}} t} K(t, r) d r\right)\left(\omega_{\frac{2 i+2}{2^{m+1}} t}-2 \omega_{\frac{2 i+1}{2^{m+1}} t}+\omega_{\frac{2 i}{2^{m+1}} t}\right) .
\end{aligned}
$$

Since Brownian motion has independent increments, we have

$$
\left\|B_{t}^{(m+1)}-B_{t}^{(m)}\right\|_{2}^{2}=\left(\frac{2^{m}}{t}\right) \sum_{i=0}^{2^{m}-1}\left(\int_{\frac{2 i+1}{2^{m+1}} t}^{\frac{2 i+2}{2^{m+1}} t} K(t, r) d r-\int_{\frac{2 i}{2^{m+1}} t}^{\frac{2 i+1}{2^{m+1}} t} K(t, r) d r\right)^{2} .
$$

Step 2. In this step, we further simplify our problem using a rather simple observation. By change of variables, for each $i \in\left\{0, \cdots, 2^{m}-1\right\}$,

$$
\begin{aligned}
M_{i} & :=\int_{\frac{2 i+1}{2^{m+1}} t}^{\frac{2 i+2}{2^{m+1}} t} K(t, r) d r-\int_{\frac{2 i}{2^{m+1}} t}^{\frac{2 i+1}{2^{m+1}} t} K(t, r) d r \\
& =\int_{\frac{2 i}{2^{m+1}} t}^{\frac{2 i+1}{2^{m+1}} t} K\left(t, s+\frac{t}{2^{m+1}}\right)-K(t, s) d s .
\end{aligned}
$$

Using the definition of $K$ and change of variables, we observe that for all $\alpha \in(0, t-s)$,

$$
\begin{aligned}
K(t, s+\alpha) & =c_{H}(s+\alpha)^{\frac{1}{2}-H} \int_{s+\alpha}^{t}(u-s-\alpha)^{H-\frac{3}{2}} u^{H-\frac{1}{2}} d u \\
& =c_{H}(s+\alpha)^{\frac{1}{2}-H} \int_{s}^{t-\alpha}(v-s)^{H-\frac{3}{2}} v^{H-\frac{1}{2}}\left(\frac{v+\alpha}{v}\right)^{H-\frac{1}{2}} d v \\
& \leq c_{H} s^{\frac{1}{2}-H} \int_{s}^{t-\alpha}(v-s)^{H-\frac{3}{2}} v^{H-\frac{1}{2}} d v \\
& =K(t-\alpha, s),
\end{aligned}
$$

and hence

$$
K(t, s+\alpha)-K(t, s) \leq K(t-\alpha, s)-K(t, s) .
$$

On the other hand, for every $\alpha \in(0, r)$,

$$
\begin{aligned}
K(t, r-\alpha) & =c_{H}(r-\alpha)^{\frac{1}{2}-H} \int_{r-\alpha}^{t}(u-r+\alpha)^{H-\frac{3}{2}} u^{H-\frac{1}{2}} d u \\
& \leq c_{H}(r-\alpha)^{\frac{1}{2}-H} \int_{r}^{t+\alpha}(v-r)^{H-\frac{3}{2}} v^{H-\frac{1}{2}} d v \\
& =\left(\frac{r}{r-\alpha}\right)^{H-\frac{1}{2}} K(t+\alpha, r) .
\end{aligned}
$$


By setting $r=s+\alpha$, we deduce that

$$
\begin{aligned}
K(t, s+\alpha)-K(t, s) & =K(t, s+\alpha)-K(t, s+\alpha-\alpha) \\
& \geq K(t, s+\alpha)-\left(\frac{s+\alpha}{s}\right)^{H-\frac{1}{2}} K(t+\alpha, s+\alpha) .
\end{aligned}
$$

Now let $\alpha=\frac{t}{2^{m+1}}$ in Eqs. 17 and 18, and for all $s<t-\frac{t}{2^{m+1}}$, define

$$
\begin{aligned}
L_{i} & =\int_{\frac{2 i}{2^{m+1}} t}^{\frac{2 i+1}{2^{m+1}} t} K\left(t, s+\frac{t}{2^{m+1}}\right)-\left(\frac{s+\frac{t}{2^{m+1}}}{s}\right)^{H-\frac{1}{2}} K\left(t+\frac{t}{2^{m+1}}, s+\frac{t}{2^{m+1}}\right) d s \\
& =\int_{\frac{2 i+1}{2^{m+1}} t}^{\frac{2 i+2}{2^{m+1}} t} K(t, r)-\left(\frac{r}{r-\frac{t}{2^{m+1}}}\right)^{H-\frac{1}{2}} K\left(t+\frac{t}{2^{m+1}}, r\right) d r,
\end{aligned}
$$

and

$$
U_{i}:=\int_{\frac{2 i}{2^{m+1}} t}^{\frac{2 i+1}{2^{m+1}} t} K\left(t-\frac{t}{2^{m+1}}, s\right)-K(t, s) d s
$$

Then $L_{i} \leq M_{i} \leq U_{i}$ for each $i$, and it thus follows that $M_{i}^{2} \leq L_{i}^{2} \vee U_{i}^{2}$, which implies that

$$
\begin{aligned}
\left\|B_{t}^{(m+1)}-B_{t}^{(m)}\right\|_{2}^{2} & =\left(\frac{2^{m}}{t}\right) \sum_{i=0}^{2^{m}-1} M_{i}^{2} \\
& \leq\left(\left(\frac{2^{m}}{t}\right) \sum_{i=0}^{2^{m}-1} L_{i}^{2}\right) \vee\left(\left(\frac{2^{m}}{t}\right) \sum_{i=0}^{2^{m}-1} U_{i}^{2}\right) .
\end{aligned}
$$

Step 3. In this step, we find upper bounds for $L_{i}^{2}$ and $U_{i}^{2}$, respectively. We first find a control of

$$
\left(\frac{2^{m}}{t}\right) \sum_{i=0}^{2^{m}-1} L_{i}^{2}=\left(\frac{2^{m}}{t}\right) \sum_{i=0}^{2^{m}-1}\left(\int_{\frac{2 i+1}{2^{m+1}} t}^{\frac{2 i+2}{2^{m+1}} t} K(t, r)-\left(\frac{r}{r-\frac{t}{2^{m+1}}}\right)^{H-\frac{1}{2}} K\left(t+\frac{t}{2^{m+1}}, r\right) d r\right)^{2}
$$

For all $r \in\left(\frac{t}{2^{m+1}}, t\right)$, consider the function

$$
f_{r}(x)=\left(\frac{r}{r-x}\right)^{H-\frac{1}{2}} K(t+x, r), \quad 0 \leq x<r .
$$

Then $f_{r}(0)=K(t, r)$ and

$$
f_{r}\left(\frac{t}{2^{m+1}}\right)=r^{H-\frac{1}{2}}\left(r-\frac{t}{2^{m+1}}\right)^{\frac{1}{2}-H} K\left(t+\frac{t}{2^{m+1}}, r\right) .
$$

Therefore,

$$
L_{i}=\int_{\frac{2 i+1}{2^{m+1}} t}^{\frac{2 i+2}{2^{m+1}} t} \int_{\frac{t}{2^{m+1}}}^{0} f_{r}^{\prime}(x) d x d r
$$


We may compute the derivative of $f_{r}$, which is

$$
\begin{aligned}
f_{r}^{\prime}(x)= & r^{H-\frac{1}{2}}\left[\left(H-\frac{1}{2}\right)(r-x)^{-\frac{1}{2}-H} K(t+x, r)+(r-x)^{\frac{1}{2}-H} \partial_{1} K(t+x, r)\right] \\
= & \left(H-\frac{1}{2}\right) r^{H-\frac{1}{2}}(r-x)^{-\frac{1}{2}-H} K(t+x, r) \\
& +c_{H}(r-x)^{\frac{1}{2}-H}(t+x)^{H-\frac{1}{2}}(t+x-r)^{H-\frac{3}{2}},
\end{aligned}
$$

where $\partial_{1} K(t, s)$ denotes the partial derivative of $K$ with respect to the first variable. Denote

$$
g_{r}(x)=\left(H-\frac{1}{2}\right) r^{H-\frac{1}{2}}(r-x)^{-\frac{1}{2}-H} K(t+x, r)
$$

and

$$
h_{r}(x)=c_{H}(r-x)^{\frac{1}{2}-H}(t+x)^{H-\frac{1}{2}}(t+x-r)^{H-\frac{3}{2}} .
$$

Then for all $x<r, g_{r}(x) \geq 0$ and $h_{r}(x) \geq 0$. By Hölder's inequality, we obtain that

$$
\begin{aligned}
L_{i}^{2} & \leq \frac{t}{2^{m+1}} \int_{\frac{2 i+1}{2^{m+1}} t}^{\frac{2 i+2}{2^{m+1} t}}\left(\int_{0}^{\frac{t}{2^{m+1}}} g_{r}(x) d x+\int_{0}^{\frac{t}{2^{m+1}}} h_{r}(x) d x\right)^{2} d r \\
& \leq \frac{t}{2^{m}} \int_{\frac{2 i+1}{2^{m+1}} t}^{\frac{2 i+2}{2^{m+1}} t}\left(\int_{0}^{\frac{t}{2^{m+1}}} g_{r}(x) d x\right)^{2}+\left(\int_{0}^{\frac{t}{2^{m+1}}} h_{r}(x) d x\right)^{2} d r,
\end{aligned}
$$

and hence

$$
\left(\frac{2^{m}}{t}\right) \sum_{i=0}^{2^{m}-1} L_{i}^{2} \leq \int_{\frac{t}{2^{m+1}}}^{t}\left(\int_{0}^{\frac{t}{2^{m+1}}} g_{r}(x) d x\right)^{2}+\left(\int_{0}^{\frac{t}{2^{m+1}}} h_{r}(x) d x\right)^{2} d r .
$$

We control the integral of $g_{r}$ first. When $H>\frac{1}{2}$, since $t \leq 1$, we have $K(t, s) s^{H-\frac{1}{2}} \leq c_{H}$, i.e. $K(t, s) \leq c_{H} s^{\frac{1}{2}-H}$. Therefore, we deduce that

$$
\begin{aligned}
0 \leq \int_{0}^{\frac{t}{2^{m+1}}} g_{r}(x) d x & =\left(H-\frac{1}{2}\right) \int_{0}^{\frac{t}{2^{m+1}}} r^{H-\frac{1}{2}}(r-x)^{-\frac{1}{2}-H} K(t+x, r) d x \\
& \leq c_{H}\left(H-\frac{1}{2}\right) \int_{0}^{\frac{t}{2^{m+1}}}(r-x)^{-\frac{1}{2}-H} d x \\
& =c_{H}\left(\left(r-\frac{t}{2^{m+1}}\right)^{\frac{1}{2}-H}-r^{\frac{1}{2}-H}\right) .
\end{aligned}
$$

As $\left(r-\frac{t}{2^{m+1}}\right)^{\frac{1}{2}-H} \geq r^{\frac{1}{2}-H}$, it follows that

$$
\left(\left(r-\frac{t}{2^{m+1}}\right)^{\frac{1}{2}-H}-r^{\frac{1}{2}-H}\right)^{2} \leq\left(r-\frac{t}{2^{m+1}}\right)^{1-2 H}-r^{1-2 H} .
$$

Consequently,

$$
\left(\int_{0}^{\frac{t}{2^{m+1}}} g_{r}(x) d x\right)^{2} \leq C_{1}\left[\left(r-\frac{t}{2^{m+1}}\right)^{1-2 H}-r^{1-2 H}\right],
$$


where $C_{1}$ is a constant depending only on $H$. As for $h_{r}$, due to change of variables,

$$
\begin{aligned}
0 \leq \int_{0}^{\frac{t}{2^{m+1}}} h_{r}(x) d x & =c_{H} \int_{0}^{\frac{t}{2^{m+1}}}(r-x)^{\frac{1}{2}-H}(t+x)^{H-\frac{1}{2}}(t+x-r)^{H-\frac{3}{2}} d x \\
& \leq c_{H}(2 t)^{H-\frac{1}{2}} \int_{0}^{\frac{t}{2^{m+1}}}(r-x)^{\frac{1}{2}-H}(t+x-r)^{H-\frac{3}{2}} d x \\
& =c_{H}(2 t)^{H-\frac{1}{2}} \int_{r-\frac{t}{2^{m+1}}}^{r} y^{\frac{1}{2}-H}(t-y)^{H-\frac{3}{2}} d y \\
& \leq \frac{2 c_{H}}{H-\frac{1}{2}}\left(r-\frac{t}{2^{m+1}}\right)^{\frac{1}{2}-H}\left(\left(t+\frac{t}{2^{m+1}}-r\right)^{H-\frac{1}{2}}-(t-r)^{H-\frac{1}{2}}\right) \\
& \leq \frac{2 c_{H}}{H-\frac{1}{2}}\left(r-\frac{t}{2^{m+1}}\right)^{\frac{1}{2}-H}\left(\frac{t}{2^{m+1}}\right)^{H-\frac{1}{2}},
\end{aligned}
$$

which implies that

$$
\left(\int_{0}^{\frac{t}{2^{m+1}}} h_{r}(x) d x\right)^{2} \leq C_{2}\left(\frac{t}{2^{m+1}}\right)^{2 H-1}\left(r-\frac{t}{2^{m+1}}\right)^{1-2 H}
$$

with $C_{2}$ some constant only depending on the value of $H$. Using (20), we get that

$$
\begin{aligned}
\left(\frac{2^{m}}{t}\right) \sum_{i=0}^{2^{m}-1} L_{i}^{2} \leq & C_{1} \int_{\frac{t}{2^{m+1}}}^{t}\left(r-\frac{t}{2^{m+1}}\right)^{1-2 H}-r^{1-2 H} d r \\
& +C_{2}\left(\frac{t}{2^{m+1}}\right)^{2 H-1} \int_{\frac{t}{2^{m+1}}}^{t}\left(r-\frac{t}{2^{m+1}}\right)^{1-2 H} d r \\
= & C_{1} \frac{1}{2-2 H}\left(\left(t-\frac{t}{2^{m+1}}\right)^{2-2 H}-t^{2-2 H}+\left(\frac{t}{2^{m+1}}\right)^{2-2 H}\right) \\
& \left.+C_{2}\left(\frac{t}{2^{m+1}}\right)^{2 H-1} \frac{1}{2-2 H}\left(t-\frac{t}{2^{m+1}}\right)^{2-2 H}\right)^{2 H-1}, \\
\leq & c_{1}\left(\frac{t}{2^{m+1}}\right)^{2-2 H}+c_{2}\left(\frac{t}{2^{m+1}}\right)^{2 H},
\end{aligned}
$$

where $c_{1}$ and $c_{2}$ are two positive constants.

Next, we move onto the estimate for $U_{i}$ 's. By the definition of $U_{i}$ 's and Hölder's inequality, we have that

$$
\begin{aligned}
\left(\frac{2^{m}}{t}\right) \sum_{i=0}^{2^{m}-1} U_{i}^{2} \leq & \left(\frac{2^{m}}{t}\right) \sum_{i=0}^{2^{m}-1}\left(\frac{t}{2^{m+1}}\right) \int_{\frac{2 i}{2^{m+1}} t}^{\frac{2 i+1}{2^{m+1}} t}\left(K\left(t-\frac{t}{2^{m+1}}, s\right)-K(t, s)\right)^{2} d s \\
\leq & \frac{1}{2} \int_{0}^{t-\frac{t}{2^{m+1}}} K^{2}\left(t-\frac{t}{2^{m+1}}, s\right) d s-\int_{0}^{t-\frac{t}{2^{m+1}}} K\left(t-\frac{t}{2^{m+1}}, s\right) K(t, s) d s \\
& +\frac{1}{2} \int_{0}^{t} K^{2}(t, s) d s \\
= & \frac{1}{2}\left(\frac{t}{2^{m+1}}\right)^{2 H}
\end{aligned}
$$


Step 4. In this step, we complete our proof using the above estimates. It follows from Eq. 19, 23 and 24 that

$$
\begin{aligned}
\left\|B_{t}^{(m+1)}-B_{t}^{(m)}\right\|_{2}^{2} & \leq\left(c_{1}\left(\frac{t}{2^{m+1}}\right)^{2-2 H}+c_{2}\left(\frac{t}{2^{m+1}}\right)^{2 H-1}\right) \vee \frac{1}{2}\left(\frac{t}{2^{m+1}}\right)^{2 H} \\
& \leq c_{3}\left(\frac{t}{2^{m+1}}\right)^{2-2 H}+c_{4}\left(\frac{t}{2^{m+1}}\right)^{2 H-1}
\end{aligned}
$$

where $c_{3}$ and $c_{4}$ are some constants.

Therefore, for any $\lambda>0$, it holds that

$$
\begin{aligned}
c_{p, r}\left(\left|B_{t}^{(m+1)}-B_{t}^{(m)}\right|>\lambda\right) & \leq C_{r, p} \lambda^{-2}\left\|B_{t}^{(m+1)}-B_{t}^{(m)}\right\|_{2}^{2} \\
& \leq C_{r, p} \lambda^{-2}\left(c_{3}\left(\frac{t}{2^{m+1}}\right)^{2-2 H}+c_{4}\left(\frac{t}{2^{m+1}}\right)^{2 H-1}\right) .
\end{aligned}
$$

Set $\lambda=2^{-m \delta}$, then as $t \leq 1$,

$$
c_{p, r}\left(\left|B_{t}^{(m+1)}-B_{t}^{(m)}\right|>\frac{1}{2^{m \delta}}\right) \leq C_{r, p, H}\left(\frac{1}{2^{2 m(1-H-\delta)}}+\frac{1}{2^{2 m\left(H-\frac{1}{2}-\delta\right)}}\right),
$$

where $C_{r, p, H}$ is a suitable constant depending only on $r, p$ and $H$. Hence, if we choose $\delta$ small enough such that $0<\delta<(1-H) \wedge\left(H-\frac{1}{2}\right)$, then

$$
\sum_{m=1}^{\infty} c_{p, r}\left(\left|B_{t}^{(m+1)}-B_{t}^{(m)}\right|>\frac{1}{2^{m \delta}}\right)<\infty,
$$

which implies that $\left(B_{t}^{(m)}\right)_{m \in \mathbb{N}}$ converges $(p, r)$-quasi-surely to some random variable $\tilde{B}_{t}$ as $m$ tends to infinity by the first Borel-Cantelli lemma. One can show that $\left(B_{t}^{(m)}\right)_{m \in \mathbb{N}}$ converges in $\mathbb{D}_{r}^{p}$ to $B_{t}$ (see e.g. [29] for a proof), and

$$
D B_{t}(s)=K(t, s) 1_{[0, t]}(s),
$$

with all higher order Malliavin derivatives of $B_{t}$ equal to zero. Now we can easily prove that there exists a subsequence $\left(B_{t}^{\left(m_{k}\right)}\right)_{k \in \mathbb{N}}$ converging $(p, r)$-quasi-surely by choosing this sequence to be such that (for example by applying Hölder's inequality)

$$
\left\|\left(B_{t}^{\left(m_{k+1}\right)}-B_{t}^{\left(m_{k}\right)}\right)^{2}\right\|_{\mathbb{D}_{r}^{p}} \leq \frac{1}{2^{k+1}},
$$

and applying the first Borel-Cantelli lemma as before. If for $\omega \in \boldsymbol{W}$, there are infinitely many $k$ 's such that $\left|B_{t}^{\left(m_{k+1}\right)}(\omega)-B_{t}^{\left(m_{k}\right)}(\omega)\right|>1$, then $\left(B_{t}^{\left(m_{k}\right)}(\omega)\right)_{k \in \mathbb{N}}$ is not Cauchy. Therefore, by Chebyshev's inequality,

$$
\begin{aligned}
& \sum_{k=0}^{\infty} c_{p, r}\left\{\omega:\left|B_{t}^{\left(m_{k+1}\right)}(\omega)-B_{t}^{\left(m_{k}\right)}(\omega)\right|>1\right\} \\
= & \sum_{k=0}^{\infty} c_{p, r}\left\{\omega:\left(B_{t}^{\left(m_{k+1}\right)}(\omega)-B_{t}^{\left(m_{k}\right)}(\omega)\right)^{2}>1\right\} \\
\leq & \sum_{k=0}^{\infty} \frac{1}{2^{k+1}}<\infty
\end{aligned}
$$


and hence by the first Borel-Cantelli lemma,

$$
c_{p, r}\left\{\left|B_{t}^{\left(m_{k+1}\right)}-B_{t}^{\left(m_{k}\right)}\right|>1 \text { infinitely often }\right\}=0 .
$$

As a consequence, $\left(B_{t}^{\left(m_{k}\right)}\right)_{k \in \mathbb{N}}$ converges to $B_{t}$ apart from on a slim set, and the uniqueness of limit forces its limit to be $\tilde{B}_{t}$, which implies $B_{t}=\tilde{B}_{t}$ q.s.

From now on, we work with the modification of $B$ which is the $(p, r)$-quasi-sure limit of the approximations $B^{(m)}$.

\section{Exponential Tightness of the Approximation Sequence}

For each fixed $t, B_{t}$ is quasi-surely defined (with $B_{0}(\omega)=0$ for all $\omega \in \boldsymbol{W}$ ). We define a map $X^{(m)}: \boldsymbol{W} \rightarrow \boldsymbol{W}$ by

$$
X^{(m)}(\omega)(t):=B_{t_{k-1}^{m}}(\omega)+2^{m}\left(t-t_{k-1}^{m}\right)\left(B_{t_{k}^{m}}(\omega)-B_{t_{k-1}^{m}}(\omega)\right), \quad \forall t_{k-1}^{m} \leq t \leq t_{k}^{m},
$$

where $t_{k}^{m}=\frac{k}{2^{m}}$. Then $X^{(m)}$ is $(p, r)$-quasi-surely defined as it is a linear interpolation of finitely many $B_{t_{k}^{m}}$ 's for all $p$ and $r$. For each $m$, let $X^{\varepsilon,(m)}$ be the scaled map, which is defined as $X^{\varepsilon,(m)}(\omega)=X^{(m)}(\varepsilon \omega)$. As $B_{t}$ is the limit of linear combinations of $\omega_{t}$ 's, it follows that $X^{\varepsilon,(m)}(\omega)=\varepsilon X^{(m)}(\omega)$.

Our goal is to show that the sequence $\left(X^{(m)}\right)_{m \in \mathbb{N}}$ converges to some $X$ quasi-surely, which implies that $\left(X^{\varepsilon,(m)}\right)_{m \in \mathbb{N}}$ converges to $X^{\varepsilon}$ quasi-surely, where the scaled map $X^{\varepsilon}$ is given by $X^{\varepsilon}(\omega)=X(\varepsilon \omega)=\varepsilon X(\omega)$. Moreover, the fact that the sequence of scaled maps $\left(X^{\varepsilon,(m)}\right)_{m \in \mathbb{N}}$ converges exponentially fast will be revealed in the proof as well. Since $X^{\varepsilon}$ is quasi-surely defined with exponentially good approximations $\left(X^{\varepsilon,(m)}\right)_{m \in \mathbb{N}}$, we may apply the result from the LDP theory to conclude the final result.

We will need the following estimate from the rough path analysis, which is contained in [31] (see Proposition 4.1.1 on page 62 or equation (4.15) on page 64). Here, we adapt the result to our case and state it as the following:

Proposition 9 Let $u$ and $w$ be two continuous paths in a Banach space. Then for any $q>1$ and $\gamma>q-1$, there exists a constant $C_{q, \gamma}$ depending only on $q$ and $\gamma$ such that

$$
\sup _{D} \sum_{l}\left|u_{t_{l-1}, t_{l}}-w_{t_{l-1}, t_{l}}\right|^{q} \leq C_{q, \gamma} \sum_{n=1}^{\infty} n^{\gamma} \sum_{k=1}^{2^{n}}\left|u_{t_{k-1}^{n}, t_{k}^{n}}-w_{t_{k-1}^{n}, t_{k}^{n}}\right|^{q},
$$

where the supremum is taken over all finite partitions $D$ of $[0,1], t_{l}^{n}=\frac{l}{2^{n}}$ for $n=1,2, \cdots$, $l=0, \cdots, 2^{n}$, and $u_{s, t}=u_{t}-u_{s}$ is the increment of path $u$.

Together with Proposition 3, the above estimate allows us to simplify our problem by controlling the $L^{2}$-norm of Gaussian processes instead of capacities.

Theorem 10 For $r \in \mathbb{N}$ and $1<p<\infty,\left(X^{(m)}\right)_{m \in \mathbb{N}}$ converges $(p, r)$-quasi-surely to some limit $X$, and the scaled maps $\left(X^{\varepsilon,(m)}\right)_{m \in \mathbb{N}}$ are exponentially good approximations of $\left\{X^{\varepsilon}: \varepsilon>0\right\}$ under the capacity $c_{p, r}$. 
Proof Here we use a technique in the theory of rough paths to control the tails of $X^{(m)}$ 's, which are Gaussian. Let us first prove that the sequence $\left(X^{(m)}\right)_{m \in \mathbb{N}}$ converges uniformly $(p, r)$-quasi-surely. By using the elementary fact that

$$
\|u-w\| \leq \sup _{D}\left(\sum_{l}\left|u_{t_{l-1}, t_{l}}-w_{t_{l-1}, t_{l}}\right|^{q}\right)^{\frac{1}{q}}
$$

for any $u, w \in \boldsymbol{W}$ and for any $q>1$, where the supremum is taken over all possible finite partitions of $[0,1]$, and $u_{s, t}=u_{t}-u_{s}$, together with Proposition 9, we obtain that

$$
\|u-w\|^{q} \leq C_{q, \gamma} \sum_{n=1}^{\infty} n^{\gamma} \sum_{k=1}^{2^{n}}\left|u_{t_{k-1}^{n}, t_{k}^{n}}-w_{t_{k-1}^{n}, t_{k}^{n}}\right|^{q}
$$

for $\gamma>q-1$, where $C_{q, \gamma}$ is a constant depending on $q$ and $\gamma$, and $t_{k}^{n}=\frac{k}{2^{n}}$. We will apply the above estimate to $X^{(m)}$ to obtain an upper bound of

$$
I_{m}(\lambda):=c_{p, r}\left(\left\|X^{(m+1)}-X^{(m)}\right\|>\lambda\right),
$$

where $\lambda>0$. Since $c_{p, r}$ is increasing in $p$, we shall assume that $p>2$. By monotonicity and sub-additivity properties of capacity, we obtain that for $\theta>0$,

$$
\begin{aligned}
I_{m}(\lambda) & \leq c_{p, r}\left(C_{q, \gamma} \sum_{n=1}^{\infty} n^{\gamma} \sum_{k=1}^{2^{n}}\left|X_{t_{k-1}^{n}, t_{k}^{n}}^{(m+1)}-X_{t_{k-1}^{n}, t_{k}^{n}}^{(m)}\right|^{q}>\lambda^{q}\right) \\
& =c_{p, r}\left(\sum_{n=1}^{\infty} n^{\gamma} \sum_{k=1}^{2^{n}}\left|X_{t_{k-1}^{n}, t_{k}^{n}}^{(m+1)}-X_{t_{k-1}^{n}, t_{k}^{n}}^{(m)}\right|^{q}>C_{q, \gamma}^{-1} C_{\theta, \gamma} \sum_{n=1}^{\infty} n^{\gamma} \frac{\lambda^{q}}{2^{n \theta}}\right) \\
& \leq \sum_{n=1}^{\infty} c_{p, r}\left(\sum_{k=1}^{2^{n}}\left|X_{t_{k-1}^{n}, t_{k}^{n}}^{(m+1)}-X_{t_{k-1}^{n}, t_{k}^{n}}^{(m)}\right|^{q}>C_{q, \gamma}^{-1} C_{\theta, \gamma} \frac{\lambda^{q}}{2^{n \theta}}\right) \\
& \leq \sum_{n=1}^{\infty} \sum_{k=1}^{2^{n}} c_{p, r}\left(\left|X_{t_{k-1}^{n}, t_{k}^{n}}^{(m+1)}-X_{t_{k-1}^{n}, t_{k}^{n}}^{(m)}\right|>C_{q, \gamma}^{-\frac{1}{q}} C_{\theta, \gamma}^{\frac{1}{q}} \frac{\lambda}{2^{\frac{n(1+\theta)}{q}}}\right),
\end{aligned}
$$

where $C_{\theta, \gamma}=\left(\sum_{n=1}^{\infty} \frac{n^{\gamma}}{2^{n \theta}}\right)^{-1}$. We introduce a new parameter $N$, whose value is to be determined at the end of our proof, and consider

$$
c_{p, r}\left(\left|X_{t_{k-1}^{n}, t_{k}^{n}}^{(m+1)}-X_{t_{k-1}^{n}, t_{k}^{n}}^{(m)}\right|^{2 N}>C_{q, \gamma}^{-\frac{2 N}{q}} C_{\theta, \gamma}^{\frac{2 N}{q}} \frac{\lambda^{2 N}}{2^{\frac{2 n N(1+\theta)}{q}}}\right) .
$$

Notice that when $n \leq m, X_{t_{k}^{n}}^{(m)}=B_{t_{2^{m-n} n_{k}}^{m}}$. Since $t_{2^{m-n} k}^{m}=t_{2^{m+1-n} k}^{m+1}$, we have that $X_{t_{k-1}^{n}, t_{k}^{n}}^{(m+1)}=$ $X_{t_{k-1}^{n}, t_{k}^{n}}^{(m)}$. By Chebyshev's inequality, we obtain that

$$
\begin{aligned}
& c_{p, r}\left(\left|X_{t_{k-1}^{n}, t_{k}^{n}}^{(m+1)}-X_{t_{k-1}^{n}, t_{k}^{n}}^{(m)}\right|^{2 N}>C_{q, \gamma, \theta, N} \frac{\lambda^{2 N}}{2^{\frac{2 n N(1+\theta)}{q}}}\right) \\
\leq & C_{q, \gamma, \theta, N}^{-1} \lambda^{-2 N} 2^{\frac{2 n N(1+\theta)}{q}}\left\|\left|X_{t_{k-1}^{n}, t_{k}^{n}}^{(m+1)}-X_{t_{k-1}^{n}, t_{k}^{n}}^{(m)}\right|^{2 N}\right\|_{\mathbb{D}_{r}^{p}},
\end{aligned}
$$


where $C_{q, \gamma, \theta, N}=C_{q, \gamma}^{-\frac{2 N}{q}} C_{\theta, \gamma}^{\frac{2 N}{q}}$ is a constant. Now by Proposition 3, with $\left|X_{t_{k-1}^{n}, t_{k}^{n}}^{(m+1)}-X_{t_{k-1}^{n}, t_{k}^{n}}^{(m)}\right|^{2 N}$ a polynomial functional of degree $2 N$, and $N \geq \frac{r}{2}$, we have that

$$
\|\| D^{l}\left(\left|X_{t_{k-1}^{n}, t_{k}^{n}}^{(m+1)}-X_{t_{k-1}^{n}, t_{k}^{n}}^{(m)}\right|^{2 N}\right)\left\|_{\mathscr{H}^{\otimes} \otimes l}\right\|\left\|_{p} \leq(2 N+1)(p-1)^{\frac{N}{2}}\right\|\left\|D^{l}\left(\left|X_{t_{k-1}^{n}, t_{k}^{n}}^{(m+1)}-X_{t_{k-1}^{n}, t_{k}^{n}}^{(m)}\right|^{2 N}\right)\right\|_{\mathscr{H} \otimes l} \|_{2},
$$

and

$$
\|\| D^{l}\left(\left|X_{t_{k-1}^{n}, t_{k}^{n}}^{(m+1)}-X_{t_{k-1}^{n}, t_{k}^{n}}^{(m)}\right|^{2 N}\right)\left\|_{\mathscr{H} \otimes l}\right\|_{2} \leq(2 N)^{\frac{r}{2}}\left\|\left|X_{t_{k-1}^{n}, t_{k}^{n}}^{(m+1)}-X_{t_{k-1}^{n}, t_{k}^{n}}^{(m)}\right|^{2 N}\right\|_{2}
$$

for any $0 \leq l \leq r$. The above two inequalities imply that

$$
\left\|\left|X_{t_{k-1}^{n}, t_{k}^{n}}^{(m+1)}-X_{t_{k-1}^{n}, t_{k}^{n}}^{(m)}\right|^{2 N}\right\|_{\mathbb{D}_{r}^{p}} \leq(r+1)(2 N+1)(p-1)^{\frac{N}{2}}(2 N)^{\frac{r}{2}}\left\|\left|X_{t_{k-1}^{n}, t_{k}^{n}}^{(m+1)}-X_{t_{k-1}^{n}, t_{k}^{n}}^{(m)}\right|^{2 N}\right\|_{2} .
$$

For $N=1,2, \cdots, f(x)=x^{2 N}$ is convex, so by Jensen's inequality,

$$
f(x+y) \leq 2^{2 N-1}(f(x)+f(y)),
$$

and hence

$$
\left|X_{t_{k-1}^{n}, t_{k}^{n}}^{(m+1)}-X_{t_{k-1}^{n}, t_{k}^{n}}^{(m)}\right|^{2 N} \leq 2^{2 N-1}\left(\left|X_{t_{k-1}^{n}, t_{k}^{n}}^{(m+1)}\right|^{2 N}+\left|X_{t_{k-1}^{n}, t_{k}^{n}}^{(m)}\right|^{2 N}\right)
$$

Therefore, it suffices to estimate $\left\|\left|X_{t_{k-1}^{n}, t_{k}^{n}}^{(m)}\right|^{2 N}\right\|_{2}$. By definition, if $t_{l-1}^{m} \leq t_{k-1}^{n}<t_{k}^{n} \leq t_{l}^{m}$ for some $l$, then

$$
X_{t_{k-1}^{n}, t_{k}^{n}}^{(m)}=2^{m}\left(t_{k}^{n}-t_{k-1}^{n}\right)\left(B_{t_{l}^{m}}-B_{t_{l-1}^{m}}\right) .
$$

Hence, by Proposition 3,

$$
\begin{aligned}
\left\|\left|X_{t_{k-1}^{n}, t_{k}^{n}}^{(m)}\right|^{2 N}\right\|_{2} & =\left\|X_{t_{k-1}^{n}, t_{k}^{n}}^{(m)}\right\|_{4 N}^{2 N} \\
& \leq 2^{2 N}(4 N-1)^{N}\left\|X_{t_{k-1}^{n}, t_{k}^{n}}^{(m)}\right\|_{2}^{2 N} \\
& =2^{2 N}(4 N-1)^{N} \mathbb{E}\left[\left(2^{m}\left(t_{k}^{n}-t_{k-1}^{n}\right)\left(B_{t_{l}^{m}}-B_{t_{l-1}^{m}}\right)\right)^{2}\right]^{N} \\
& =2^{2 N}(4 N-1)^{N} \frac{2^{2 m N(1-H)}}{2^{2 n N}} .
\end{aligned}
$$

It thus implies that

$$
\begin{aligned}
\left\|\left|X_{t_{k-1}^{n}, t_{k}^{n}}^{(m+1)}-X_{t_{k-1}^{n}, t_{k}^{n}}^{(m)}\right|^{2 N}\right\|_{2} & \leq 2^{2 N-1}\left(\left\|\left|X_{t_{k-1}^{n}, t_{k}^{n}}^{(m+1)}\right|^{2 N}\right\|_{2}+\left\|\left|X_{t_{k-1}^{n}, t_{k}^{n}}^{(m)}\right|^{2 N}\right\|_{2}\right) \\
& \leq 2^{4 N-1}(4 N-1)^{N}\left(\frac{2^{2(m+1) N(1-H)}}{2^{2 n N}}+\frac{2^{2 m N(1-H)}}{2^{2 n N}}\right) \\
& =C_{N, H} \frac{2^{2 m N(1-H)}}{2^{2 n N}},
\end{aligned}
$$


where $C_{N, H}=2^{4 N-1}(4 N-1)^{N}\left(1+2^{2 N(1-H)}\right)$ is a constant depending only on $N$ and $H$. We may conclude from Eqs. 29 and 30 that

$$
\left\|\left|X_{t_{k-1}^{n}, t_{k}^{n}}^{(m+1)}-X_{t_{k-1}^{n}, t_{k}^{n}}^{(m)}\right|^{2 N}\right\|_{\mathbb{D}_{r}^{p}} \leq C_{r, p, N, H} \frac{2^{2 m N(1-H)}}{2^{2 n N}}
$$

for $n>m$, where

$$
C_{r, p, N, H}=(r+1)(2 N+1)(p-1)^{\frac{N}{2}}(2 N)^{\frac{r}{2}} C_{N, H}
$$

depends on $r, p, N$ and $H$. Plugging (31) into (28), we obtain that

$$
c_{p, r}\left(\left|X_{t_{k-1}^{n}, t_{k}^{n}}^{(m+1)}-X_{t_{k-1}^{n}, t_{k}^{n}}^{(m)}\right|^{2 N}>C_{q, \gamma, \theta} \frac{\lambda^{2 N}}{2^{\frac{2 n N(1+\theta)}{q}}}\right) \leq C_{q, \gamma, \theta, N}^{-1} C_{r, p, N, H} \lambda^{-2 N} \frac{2^{2 m N(1-H)}}{2^{2 n N\left(1-\frac{1+\theta}{q}\right)}} .
$$

Therefore, according to Eqs. 26, 27 and 33,

$$
\begin{aligned}
I_{m}(\lambda) & \leq \sum_{n=1}^{\infty} \sum_{k=1}^{2^{n}} c_{p, r}\left(\left|X_{t_{k-1}^{n}, t_{k}^{n}}^{(m+1)}-X_{t_{k-1}^{n}, t_{k}^{n}}^{(m)}\right|^{2 N}>C_{q, \gamma, \theta, N} \frac{\lambda^{2 N}}{2^{\frac{2 n N(1+\theta)}{q}}}\right) \\
& \leq \sum_{n=m+1}^{\infty} \sum_{k=1}^{2^{n}} C_{q, \gamma, \theta, N}^{-1} C_{r, p, N, H} \lambda^{-2 N} \frac{2^{2 m N(1-H)}}{2^{2 n N\left(1-\frac{1+\theta}{q}\right)}} \\
& =C_{q, \gamma, \theta, N}^{-1} C_{r, p, N, H} \lambda^{-2 N} \sum_{n=m+1}^{\infty} \frac{2^{2 m N(1-H)}}{2^{n\left(2 N\left(1-\frac{1+\theta}{q}\right)-1\right)}} \\
& =C_{q, \gamma, \theta, N}^{-1} C_{r, p, N, H} \lambda^{-2 N} \frac{1}{2^{m\left(2 N\left(H-\frac{1+\theta}{q}\right)-1\right)}} \sum_{k=1}^{\infty} \frac{1}{2^{k\left(2 N\left(1-\frac{1+\theta}{q}\right)-1\right)}}
\end{aligned}
$$

Since $2 N\left(1-\frac{1+\theta}{q}\right)-1>2 N\left(H-\frac{1+\theta}{q}\right)-1$, the above series converges as long as $2 N\left(H-\frac{1+\theta}{q}\right)-1>0$, which means that we need $q H-\frac{q}{2 N}-1>0$ for some integer $N$. Therefore, if we choose $q>\left(H-\frac{1}{2}\right)^{-1}$, and $\theta \in\left(0, q H-\frac{q}{2 N}-1\right)$ then the above series converges. As a consequence, we thus have

$$
I_{m}(\lambda) \leq C_{q, \gamma, \theta, N}^{\prime} C_{r, p, N, H} \frac{\lambda^{-2 N}}{2^{m\left(2 N\left(H-\frac{1+\theta}{q}\right)-1\right)}}
$$

for every $m=1,2, \cdots$, where

$$
\begin{aligned}
C_{q, \gamma, \theta, N}^{\prime} & =C_{q, \gamma, \theta, N}^{-1} \sum_{k=1}^{\infty} \frac{1}{2^{k\left(2 N\left(1-\frac{1+\theta}{q}\right)-1\right)}} \\
& \leq C_{q, \gamma, \theta, N}^{-1} \frac{1}{2^{2\left(1-\frac{1+\theta}{q}\right)-1}-1} \\
& =C_{q, \gamma, \theta, N}^{-1} C_{q, \theta}
\end{aligned}
$$


with $C_{q, \theta}=\left(2^{2\left(1-\frac{1+\theta}{q}\right)-1}-1\right)^{-1}$. From Eq. 34, we may deduce that

$$
I_{m}(\lambda) \leq C_{q, \gamma, \theta, N}^{-1} C_{q, \theta} C_{r, p, N, H} \frac{\lambda^{-2 N}}{2^{m\left(2 N\left(H-\frac{1+\theta}{q}\right)-1\right)}} .
$$

Applying the same argument as in the previous theorem, we see that the problem may be reduced to proving that for a suitable positive $\delta>0$,

$$
\sum_{m=1}^{\infty} I_{m}\left(\frac{1}{2^{m \delta}}\right)<\infty
$$

Then by the first Borel-Cantelli lemma for capacity, we obtain the quasi-sure convergence for $\left(X^{(m)}\right)_{m \in \mathbb{N}}$. Since

$$
I_{m}\left(\frac{1}{2^{m \delta}}\right) \leq C_{q, \gamma, \theta, N}^{-1} C_{q, \theta} C_{r, p, N, H} \frac{1}{2^{m\left(2 N\left(H-\frac{1+\theta}{q}-\delta\right)-1\right)}},
$$

so the series in Eq. 36 converges as long as we choose $\delta$ such that $\delta<H-\frac{1+\theta}{q}-\frac{1}{2 N}$, which must exist as we have chosen $q$ and $\theta$ such that $2 N\left(H-\frac{1+\theta}{q}\right)-1>0$ for some $N \in \mathbb{N}$. Thus, the convergence of the series in Eq. 36 implies the convergence of $\left(X^{(m)}\right)_{m \in \mathbb{N}}$. Denote its limit by $X$, then $X$ is defined quasi-surely on $\boldsymbol{W}$.

Next, we prove the sequence $\left\{X^{\varepsilon,(m)}: m \geq 1, \varepsilon>0\right\}$ converges to $\left\{X^{\varepsilon}: \varepsilon>0\right\}$ exponentially fast with respect to the capacity $c_{p, r}$, that is,

$$
\lim _{m \rightarrow \infty} \limsup _{\varepsilon \rightarrow 0} \varepsilon^{2} \log c_{p, r}\left\{\omega:\left\|X^{\varepsilon,(m)}(\omega)-X^{\varepsilon}(\omega)\right\|>\lambda\right\}=-\infty .
$$

To this end, we shall use a similar argument as in the proof above. By the sub-additivity of capacity, for $\alpha>0$,

$$
\begin{aligned}
& c_{p, r}\left\{\omega:\left\|X^{\varepsilon,(m)}(\omega), X^{\varepsilon}(\omega)\right\|>\lambda\right\} \\
\leq & c_{p, r}\left\{\omega: \sum_{k=m}^{\infty}\left\|X^{\varepsilon,(k)}(\omega), X^{\varepsilon,(k+1)}(\omega)\right\|>\lambda\right\} \\
= & c_{p, r}\left\{\omega: \sum_{k=m}^{\infty}\left\|X^{\varepsilon,(k)}(\omega), X^{\varepsilon,(k+1)}(\omega)\right\|>C_{\alpha} \sum_{k=m}^{\infty} \frac{\lambda}{2^{(k-m) \alpha}}\right\} \\
\leq & \sum_{k=m}^{\infty} c_{p, r}\left\{\omega:\left\|X^{(k)}(\omega), X^{(k+1)}(\omega)\right\|>C_{\alpha} \varepsilon^{-1} \frac{\lambda}{2^{(k-m) \alpha}}\right\} \\
= & \sum_{k=m}^{\infty} I_{k}\left(\frac{\lambda C_{\alpha}}{2^{(k-m) \alpha} \varepsilon}\right),
\end{aligned}
$$

where we have used the notations in Eq. 25, and $C_{\alpha}=\left(\sum_{i=0}^{\infty} \frac{1}{2^{i \alpha}}\right)^{-1}$ is some positive constant depending only on $\alpha$. Recall that up to now, the only assumption on $N$ is that 
$N \geq \frac{r}{2}$, and now we shall pick up a suitable $N$ to show that the convergence of $\left(X^{\varepsilon,(m)}\right)_{m \in \mathbb{N}}$ is exponentially fast. By Eq. 35,

$$
\begin{aligned}
I_{k}\left(\frac{\lambda C_{\alpha}}{2^{(k-m) \alpha} \varepsilon}\right) & \leq C_{q, \gamma, \theta, N}^{-1} C_{r, p, N, H} C_{q, \theta} \frac{1}{2^{k\left(2 N\left(H-\frac{1+\theta}{q}-\alpha\right)-1\right)}} \frac{1}{2^{2 N m \alpha}} \frac{\varepsilon^{2 N}}{\lambda^{2 N} C_{\alpha}^{2 N}} \\
& =C_{q, \gamma, \theta, N}^{-1} C_{r, p, N, H} C_{q, \theta} C_{\alpha}^{-2 N} \frac{1}{2^{k \beta}} \frac{1}{2^{2 N m \alpha}} \frac{\varepsilon^{2 N}}{\lambda^{2 N}},
\end{aligned}
$$

where $\beta=2 N\left(H-\frac{1+\theta}{q}-\alpha\right)-1$. As $C_{q, \gamma, \theta, N}=C_{q, \gamma}^{-\frac{2 N}{q}} C_{\theta, \gamma}^{\frac{2 N}{q}}$, where $C_{r, p, N, H}$ is given as in Eq. 32 with $C_{N, H}=2^{4 N-1}(4 N-1)^{N}\left(1+2^{2 N(1-H)}\right)$, we have that

$$
\begin{aligned}
& C_{q, \gamma, \theta, N}^{-1} C_{r, p, N, H} \\
= & C_{q, \gamma}^{\frac{2 N}{q}} C_{\theta, \gamma}^{-\frac{2 N}{q}}(r+1)(2 N+1)(p-1)^{\frac{N}{2}}(2 N)^{\frac{r}{2}} 2^{4 N-1}(4 N-1)^{N}\left(1+2^{2 N(1-H)}\right) \\
\leq & (r+1)(2 N+1)(2 N)^{\frac{r}{2}}\left(16 C_{q, \gamma}^{\frac{2}{q}} C_{\theta, \gamma}^{-\frac{2}{q}}(p-1)^{\frac{1}{2}}\right)^{N}(4 N)^{N} 2^{2 N(1-H)} \\
:= & P_{r}(N) C_{q, \gamma, \theta, p, H}^{N} N^{N},
\end{aligned}
$$

where

$$
P_{r}(N)=(r+1)(2 N+1)(2 N)^{\frac{r}{2}}
$$

is a polynomial of $N$ depending only on $r$, and $C_{q, \gamma, \theta, p, H}=64 C_{q, \gamma}^{\frac{2}{q}} C_{\theta, \gamma}^{-\frac{2}{q}}(p-1)^{\frac{1}{2}} 2^{2(1-H)}$ is a constant. If we set $\alpha$ such that $\alpha<H-\frac{1+\theta}{q}-\frac{1}{2 N}$, then $\beta>0$. Together with Eqs. 38 and 39 , we obtain that

$$
\begin{aligned}
\sum_{k=m}^{\infty} I_{k}\left(\frac{\lambda C_{\alpha}}{2^{(k-m) \alpha} \varepsilon}\right) & \leq P_{r}(N) C_{q, \gamma, \theta, p, H}^{N} N^{N} C_{q, \theta} C_{\alpha}^{-2 N} \frac{1}{2^{2 N m \alpha}} \frac{\varepsilon^{2 N}}{\lambda^{2 N}} \sum_{k=m}^{\infty} \frac{1}{2^{k \beta}} \\
& =P_{r}(N) C_{q, \gamma, \theta, p, H}^{N} N^{N} C_{q, \theta} C_{\alpha}^{-2 N} \lambda^{-2 N} \varepsilon^{2 N} \sum_{k=0}^{\infty} \frac{1}{2^{k \beta}} \frac{1}{2^{m(2 N \alpha+\beta)}} \\
& =P_{r, q, \theta, \beta}(N) C_{q, \gamma, \theta, p, H, \alpha}^{N} \lambda^{-2 N} \varepsilon^{2 N} N^{N} \frac{1}{2^{m\left(2 N\left(H-\frac{1+\theta}{q}\right)-1\right)}},
\end{aligned}
$$

where $C_{q, \gamma, \theta, p, H, \alpha}=C_{q, \gamma, \theta, p, H} C_{\alpha}^{-2}$ and $P_{r, q, \theta, \beta}(N)=C_{q, \theta}\left(\sum_{k=0}^{\infty} \frac{1}{2^{k \beta}}\right) P_{r}(N)$. According to Eq. 37, it holds that

$$
\begin{aligned}
& \varepsilon^{2} \log c_{p, r}\left\{\omega:\left\|X^{\varepsilon,(m)}(\omega), X^{\varepsilon}(\omega)\right\|>\lambda\right\} \\
\leq & \varepsilon^{2} \log P_{r, q, \theta, \beta}(N)+\varepsilon^{2} N \log C_{q, \gamma, \theta, p, H, \alpha} \\
& +\varepsilon^{2} N \log \left(\frac{\varepsilon^{2} N}{\lambda^{2}}\right)-\varepsilon^{2}\left(2 N\left(H-\frac{1+\theta}{q}\right)-1\right) m \log 2 .
\end{aligned}
$$


For $\varepsilon$ small enough, choose $N=\left\lfloor\varepsilon^{-2}\right\rfloor$. Then since $P_{r, q, \theta, \beta}(N)$ is a polynomial of $N$, it holds that

$$
\limsup _{\varepsilon \rightarrow 0} \varepsilon^{2} \log c_{p, r}\left\{\omega:\left\|X^{\varepsilon,(m)}(\omega), X^{\varepsilon}(\omega)\right\|>\lambda\right\} \leq \log C-2\left(H-\frac{1+\theta}{q}\right) m \log 2,
$$

where $C=C_{q, \gamma, \theta, p, H, \alpha} \lambda^{-2}$ is a constant. Therefore, as $H>\frac{1+\theta}{q}$,

$$
\lim _{m \rightarrow \infty} \limsup _{\varepsilon \rightarrow 0} \varepsilon^{2} \log c_{p, r}\left\{\omega:\left\|X^{\varepsilon, m}(\omega), X^{\varepsilon}(\omega)\right\|>\lambda\right\}=-\infty,
$$

which completes the proof.

\section{The Proof of the Main Result}

This section is devoted to the proof of the large deviation principles stated in the second part of the main result, Theorem 2 .

Notice that for each $m, X^{(m)}$, which is a Wiener functional on $\boldsymbol{W}$ defined $(p, r)$-quasisurely, is a linear interpolation of some Gaussian random variables, so we may consider $F_{m}: \mathbb{R}^{2^{m}+1} \rightarrow \boldsymbol{W}$, where

$$
F_{m}\left(x_{0}, \cdots x_{2^{m}}\right)(t)=x_{k-1}+2^{m}\left(t-\frac{k-1}{2^{m}}\right)\left(x_{k}-x_{k-1}\right), \quad \forall t \in\left[\frac{k-1}{2^{m}}, \frac{k}{2^{m}}\right],
$$

which maps a $\left(2^{m}+1\right)$-dimensional vector to its linear interpolation. Let us apply Varadhan's contraction principle to the maps above. As the rate function for the vector-valued Gaussian random variable $\left(B_{0}, B_{\frac{1}{2^{m}}}, \cdots, B_{\frac{k}{2^{m}}}, \cdots, B_{1}\right)$ is computable, the quasi-sure version of LDP may be established easily for $X^{(m)}$.

Proposition 11 Let $\boldsymbol{t}:=\left\{0 \leq t_{1}<t_{2}<\cdots<t_{n} \leq 1\right\}$ be a finite partition of [0,1]. Define $T^{\varepsilon}: \boldsymbol{W} \rightarrow \mathbb{R}^{n}($ for $\varepsilon>0)$ by $T^{\varepsilon}(\omega)=\boldsymbol{B}_{\boldsymbol{t}}(\varepsilon \omega)$, where

$$
\boldsymbol{B}_{\boldsymbol{t}}(\omega)=\left(B_{t_{1}}(\omega), \cdots, B_{t_{n}}(\omega)\right)
$$

is a Gaussian vector with covariance matrix $\boldsymbol{\Sigma}=\left(\sigma_{i j}\right)_{1 \leq i, j \leq n}$ and $\sigma_{i j}=\operatorname{Cov}\left(t_{i}, t_{j}\right)$. Then $\left\{T^{\varepsilon}: \varepsilon>0\right\}$ satisfies $c_{p, r}-L D P$ with the good rate function $I_{n}: \mathbb{R}^{n} \rightarrow[0, \infty]$ given by

$$
I_{n}(x)=\frac{1}{2} x^{T} \Sigma^{-1} x
$$

Proof According to Definition 1, we need to establish the upper bound and the lower bound. Since $(p, r)$-capacity is increasing in $p$ and $r$, the lower bound part follows directly from the classical LDPs for Gaussian measures, to conclude that for all open $G \subset \boldsymbol{W}$,

$$
\begin{aligned}
\limsup _{\varepsilon \rightarrow 0} \varepsilon^{2} \log c_{p, r}\left\{\omega \in \boldsymbol{W}: T^{\varepsilon}(\omega) \in G\right\} & \geq \frac{1}{p} \limsup _{\varepsilon \rightarrow 0} \varepsilon^{2} \log \mathbb{P}\left\{\omega \in \boldsymbol{W}: T^{\varepsilon}(\omega) \in G\right\} \\
& \geq-\frac{1}{p} \inf _{y \in G} I_{n}(y) .
\end{aligned}
$$


For the upper bound part, we first establish the result when $n=1$. Let $a>0$. By Chebyshev's inequality, for all $\lambda>0$,

$$
\begin{aligned}
c_{p, r}\left\{\omega: B_{t}(\varepsilon \omega)>a\right\} & =c_{p, r}\left\{\omega: e^{\lambda \varepsilon B_{t}(\omega)}>e^{\lambda a}\right\} \\
& \leq e^{-\lambda a}\left\|e^{\lambda \varepsilon B_{t}}\right\|_{\mathbb{D}_{r}^{p}} \\
& =e^{-\lambda a}\left(\sum_{l=0}^{r} \mathbb{E}\left[\left|\left\|D^{l}\left(e^{\lambda \varepsilon B_{t}}\right)\right\|_{\mathscr{H}^{\otimes l}}\right|^{p}\right]\right)^{\frac{1}{p}} .
\end{aligned}
$$

Recall that

$$
D\left(e^{\lambda \varepsilon B_{t}}\right)(s)=\lambda \varepsilon e^{\lambda \varepsilon B_{t}} K(t, s) \mathbb{1}_{(0, t)}(s),
$$

so by iteration,

$$
D^{l}\left(e^{\lambda \varepsilon B_{t}}\right)\left(s_{1}, s_{2}, \cdots, s_{l}\right)=(\lambda \varepsilon)^{l} e^{\lambda \varepsilon B_{t}}\left(K(t) \mathbb{1}_{(0, t)}\right)^{\otimes l}\left(s_{1}, s_{2}, \cdots, s_{l}\right)
$$

for all $l \leq r$, where $\left(K(t) \mathbb{1}_{(0, t)}\right)^{\otimes l}$ denotes the $l$-fold tensor product of $K(t, s) \mathbb{1}_{(0, t)}(s)$ with itself. Therefore,

$$
\begin{aligned}
\left\|D^{l}\left(e^{\lambda \varepsilon B_{t}}\right)\right\|_{\mathscr{H} \otimes l}^{2} & =(\lambda \varepsilon)^{2 l} e^{2 \lambda \varepsilon B_{t}}\left(\int_{0}^{1} K^{2}(t, s) \mathbb{1}_{(0, t)}(s) d s\right)^{l} \\
& =(\lambda \varepsilon)^{2 l} e^{2 \lambda \varepsilon B_{t}} t^{2 H l}
\end{aligned}
$$

and hence

$$
\begin{aligned}
\mathbb{E}\left[\left|\left\|D^{l}\left(e^{\lambda \varepsilon B_{t}}\right)\right\|_{\mathscr{H} \otimes l}\right|^{p}\right] & =(\lambda \varepsilon)^{l p} t^{H l p} \mathbb{E}\left[e^{\lambda \varepsilon p B_{t}}\right] \\
& =(\lambda \varepsilon)^{l p} t^{H l p} e^{\frac{(\lambda \varepsilon p)^{2} t^{2 H}}{2}} .
\end{aligned}
$$

It thus follows that

$$
\begin{aligned}
c_{p, r}\left\{\omega: B_{t}(\varepsilon \omega)>a\right\} & \leq e^{-\lambda a}\left(\sum_{l=0}^{r} \mathbb{E}\left[\left|\left\|D^{l}\left(e^{\lambda \varepsilon B_{t}}\right)\right\|_{\mathscr{H} \otimes l}\right|^{p}\right]\right)^{\frac{1}{p}} \\
& \leq e^{-\lambda a} \sum_{l=0}^{r} \mathbb{E}\left[|| D^{l}\left(e^{\lambda \varepsilon B_{t}}\right) \|\left._{\mathscr{H} \otimes l}\right|^{p}\right]^{\frac{1}{p}} \\
& =e^{\frac{(\lambda \varepsilon)^{2} p t^{2 H}}{2}-\lambda a} \sum_{l=0}^{r}\left(\lambda \varepsilon t^{H}\right)^{l}
\end{aligned}
$$

so that

$$
\varepsilon^{2} \log c_{p, r}\left\{\omega: B_{t}(\varepsilon \omega)>a\right\} \leq \frac{\lambda^{2} \varepsilon^{4} p t^{2 H}}{2}-\lambda a \varepsilon^{2}+\varepsilon^{2} \log \left(\sum_{l=0}^{r}\left(\lambda \varepsilon t^{H}\right)^{l}\right) .
$$


Setting $\lambda=\frac{a}{p \varepsilon^{2} t^{2 H}}$ so that the sum of first two terms in Eq. 41 attains its minimum, we obtain that

$$
\begin{aligned}
\varepsilon^{2} \log c_{p, r}\left\{\omega: B_{t}(\varepsilon \omega)>a\right\} & \leq-\frac{a^{2}}{2 p t^{2 H}}+\varepsilon^{2} \log \left((r+1) \max _{0 \leq l \leq r}\left(\frac{a}{\varepsilon p t^{H}}\right)^{l}\right) \\
& =-\frac{a^{2}}{2 p t^{2 H}}+\varepsilon^{2} \log (r+1)+\max _{0 \leq l \leq r} l \varepsilon^{2} \log \left(\frac{a}{\varepsilon p t^{H}}\right) .
\end{aligned}
$$

It follows that

$$
\limsup _{\varepsilon \rightarrow 0} \varepsilon^{2} \log c_{p, r}\left\{\omega: B_{t}(\varepsilon \omega)>a\right\} \leq-\frac{1}{2 p} \cdot \frac{a^{2}}{t^{2 H}}=-\frac{1}{p} \inf _{x>a} I_{1}(x),
$$

which remains true if we replace $\left\{\omega: B_{t}(\varepsilon \omega)>a\right\}$ with $\left\{\omega: B_{t}(\varepsilon \omega) \geq a\right\}$. We may deduce the similar results for $\left\{\omega: B_{t}(\varepsilon \omega)<b\right\}$ and $\left\{\omega: B_{t}(\varepsilon \omega) \leq b\right\}$ with $b<0$ by symmetry.

Now deal the case of a finite partition $\boldsymbol{t}=\left\{0 \leq t_{1} \leq \cdots \leq t_{n} \leq 1\right\}$. Then $\boldsymbol{B}_{t}(\varepsilon \omega)=$ ${ }_{\varepsilon} \boldsymbol{B}_{t}(\omega)$. Introduce an inner product $\langle\cdot, \cdot\rangle_{\boldsymbol{\Sigma}}$ on $\mathbb{R}^{n}$ :

$$
\langle\boldsymbol{x}, \boldsymbol{y}\rangle_{\boldsymbol{\Sigma}}=\boldsymbol{x}^{T} \boldsymbol{\Sigma}^{-1} \boldsymbol{y}
$$

and denote the corresponding norm by $|\cdot|_{\boldsymbol{\Sigma}}$. Notice that for any $\boldsymbol{x}=\left(x_{1}, \cdots, x_{n}\right) \in B(\boldsymbol{a}, r)$, the open ball in $\left(\mathbb{R}^{n},|\cdot| \boldsymbol{\Sigma}\right)$ with centre $\boldsymbol{a}$ and radius $r$,

$$
\langle\lambda, \boldsymbol{a}-\boldsymbol{x}\rangle_{\boldsymbol{\Sigma}} \leq|\lambda|_{\Sigma}|\boldsymbol{a}-\boldsymbol{x}|_{\Sigma} \leq r|\lambda|_{\Sigma}
$$

for all $\lambda \in \mathbb{R}^{n}$, which implies that $B(\boldsymbol{a}, r) \subset\left\{\boldsymbol{x}:\langle\boldsymbol{\lambda}, \boldsymbol{a}-\boldsymbol{x}\rangle_{\boldsymbol{\Sigma}} \leq r|\lambda|_{\boldsymbol{\Sigma}}\right\}$. Based on this observation, we may apply Chebyshev's inequality and get that

$$
\begin{aligned}
c_{p, r}\left\{\omega: \boldsymbol{B}_{\boldsymbol{t}}(\varepsilon \omega) \in B(\boldsymbol{a}, r)\right\} & \leq c_{p, r}\left\{\omega:\left\langle\lambda, \boldsymbol{a}-\boldsymbol{B}_{\boldsymbol{t}}(\varepsilon \omega)\right\rangle_{\boldsymbol{\Sigma}} \leq r|\lambda|_{\boldsymbol{\Sigma}}\right\} \\
& =c_{p, r}\left\{\omega: e^{\left\langle\lambda, \boldsymbol{B}_{t}(\varepsilon \omega)\right\rangle_{\boldsymbol{\Sigma}}} \geq e^{\langle\lambda, \boldsymbol{a}\rangle_{\boldsymbol{\Sigma}}-r|\lambda|_{\boldsymbol{\Sigma}}}\right\} \\
& \leq e^{r|\lambda|_{\boldsymbol{\Sigma}}-\langle\lambda, \boldsymbol{a}\rangle_{\boldsymbol{\Sigma}}}\left\|e^{\left\langle\lambda, \boldsymbol{B}_{t}(\varepsilon \omega)\right\rangle_{\boldsymbol{\Sigma}}}\right\|_{\mathbb{D}_{r}^{p}}
\end{aligned}
$$

for all $\lambda=\left(\lambda_{1}, \cdots, \lambda_{n}\right) \in \mathbb{R}^{n}$. By the Chain rule for Malliavin derivatives,

$$
D\left(e^{\left\langle\lambda, \boldsymbol{B}_{t}(\varepsilon \omega)\right\rangle_{\Sigma}}\right)(s)=\varepsilon e^{\left\langle\lambda, \boldsymbol{B}_{t}(\varepsilon \omega)\right\rangle_{\Sigma}}\left\langle\lambda, D \boldsymbol{B}_{t}\right\rangle_{\Sigma}(s),
$$

where $D \boldsymbol{B}_{\boldsymbol{t}}=\left(D B_{t_{1}}, \cdots, D B_{t_{n}}\right)$, and by iteration,

$$
D^{l}\left(e^{\left\langle\lambda, \boldsymbol{B}_{t}(\varepsilon \omega)\right\rangle_{\Sigma}}\right)\left(s_{1}, \cdots, s_{l}\right)=\varepsilon^{l} e^{\left\langle\lambda, \boldsymbol{B}_{t}(\varepsilon \omega)\right\rangle_{\Sigma}}\left\langle\lambda, D \boldsymbol{B}_{\boldsymbol{t}}\right\rangle_{\Sigma}^{\otimes l}\left(s_{1}, \cdots, s_{l}\right),
$$


where $\left\langle\lambda, D \boldsymbol{B}_{\boldsymbol{t}}\right\rangle_{\boldsymbol{\Sigma}}^{\otimes l}$ denotes the $l$-fold tensor product of $\left\langle\lambda, D \boldsymbol{B}_{\boldsymbol{t}}\right\rangle_{\boldsymbol{\Sigma}}$ with itself. Since $\left\langle D B_{t_{i}}, D B_{t_{j}}\right\rangle_{\mathscr{H}}=\sigma_{i j}$ for all $1 \leq i, j \leq n$, it follows that

$$
\begin{aligned}
\left\|D^{l}\left(e^{\left\langle\lambda, \boldsymbol{B}_{\boldsymbol{t}}(\varepsilon \omega)\right\rangle_{\boldsymbol{\Sigma}}}\right)\right\|_{\mathscr{H} \otimes l}^{2} & =\varepsilon^{2 l} e^{2 \varepsilon\left\langle\lambda, \boldsymbol{B}_{\boldsymbol{t}}(\omega)\right\rangle_{\boldsymbol{\Sigma}}}\left\langle\left\langle\lambda, D \boldsymbol{B}_{\boldsymbol{t}}\right\rangle_{\boldsymbol{\Sigma}},\left\langle\lambda, D \boldsymbol{B}_{\boldsymbol{t}}\right\rangle_{\boldsymbol{\Sigma}}\right\rangle_{\mathscr{H}}^{l} \\
& =\varepsilon^{2 l} e^{2 \varepsilon\left\langle\lambda, \boldsymbol{B}_{\boldsymbol{t}}(\omega)\right\rangle_{\boldsymbol{\Sigma}}}\left(\sum_{i=1}^{n}\left(\lambda^{T} \boldsymbol{\Sigma}^{-1}\right)_{i} D B_{t_{i}}, \sum_{j=1}^{n}\left(\lambda^{T} \boldsymbol{\Sigma}^{-1}\right)_{j} D B_{t_{j}}\right)_{\mathscr{H}}^{l} \\
& =\varepsilon^{2 l} e^{2 \varepsilon\left\langle\lambda, \boldsymbol{B}_{t}(\omega)\right\rangle_{\boldsymbol{\Sigma}}}\left(\sum_{1 \leq i, j \leq n}\left(\lambda^{T} \boldsymbol{\Sigma}^{-1}\right)_{i}\left(\lambda^{T} \boldsymbol{\Sigma}^{-1}\right)_{j} \sigma_{i j}\right)^{l} \\
& =\varepsilon^{2 l}|\lambda|_{\boldsymbol{\Sigma}}^{2 l} e^{2 \varepsilon\left\langle\lambda, \boldsymbol{B}_{t}(\omega)\right\rangle_{\boldsymbol{\Sigma}}},
\end{aligned}
$$

where $\left(\lambda^{T} \boldsymbol{\Sigma}^{-1}\right)_{i}$ denotes the $i$-th component of $\lambda^{T} \boldsymbol{\Sigma}^{-1}$. Thus,

$$
\begin{aligned}
\mathbb{E}\left[\left|\left\|D^{l}\left(e^{\left\langle\lambda, \boldsymbol{B}_{t}(\varepsilon \omega)\right\rangle_{\boldsymbol{\Sigma}}}\right)\right\|_{\mathscr{H} \otimes l}\right|^{p}\right] & =\varepsilon^{l p}|\lambda|_{\boldsymbol{\Sigma}}^{l p} \mathbb{E}\left[e^{\varepsilon p\left\langle\lambda, \boldsymbol{B}_{t}(\omega)\right\rangle_{\boldsymbol{\Sigma}}}\right] \\
& =\varepsilon^{l p}|\lambda|_{\boldsymbol{\Sigma}}^{l p} e^{\frac{1}{2}(\varepsilon p)^{2}\left(\boldsymbol{\Sigma}^{-1} \lambda\right)^{T} \boldsymbol{\Sigma}\left(\boldsymbol{\Sigma}^{-1} \lambda\right)} \\
& =\varepsilon^{l p}|\lambda|_{\boldsymbol{\Sigma}}^{l p} e^{\frac{1}{2}(\varepsilon p)^{2}|\lambda|_{\Sigma}^{2}},
\end{aligned}
$$

which implies that

$$
\begin{aligned}
\left\|e^{\left\langle\lambda, \boldsymbol{B}_{t}(\varepsilon \omega)\right\rangle}\right\|_{\mathbb{D}_{r}^{p}} & \leq \sum_{l=0}^{r} \mathbb{E}\left[\left|\left\|D^{l}\left(e^{\left\langle\lambda, \boldsymbol{B}_{t}(\varepsilon \omega)\right\rangle}\right)\right\|_{\mathscr{H} \otimes l}\right|^{p}\right]^{\frac{1}{p}} \\
& =\sum_{l=0}^{r} \varepsilon^{l}|\lambda|_{\Sigma}^{l} e^{\frac{1}{2} \varepsilon^{2} p|\lambda|_{\Sigma}^{2}} .
\end{aligned}
$$

Therefore, by Eq. 43,

$$
c_{p, r}\left\{\omega: \boldsymbol{B}_{\boldsymbol{t}}(\varepsilon \omega) \in B(\boldsymbol{a}, r)\right\} \leq \sum_{l=0}^{r}\left(\varepsilon|\lambda|_{\boldsymbol{\Sigma}}\right)^{l} e^{\frac{1}{2} \varepsilon^{2} p|\lambda|_{\boldsymbol{\Sigma}}^{2}+r|\lambda|_{\boldsymbol{\Sigma}}-\langle\lambda, \boldsymbol{a}\rangle_{\boldsymbol{\Sigma}}} .
$$

As a consequence, we have that

$$
\begin{aligned}
\varepsilon^{2} \log c_{p, r}\left\{\omega: \boldsymbol{B}_{\boldsymbol{t}}(\varepsilon \omega) \in B(\boldsymbol{a}, r)\right\} \leq & \frac{1}{2} \varepsilon^{4} p|\lambda|_{\boldsymbol{\Sigma}}^{2}+\varepsilon^{2} r|\lambda|_{\boldsymbol{\Sigma}}-\varepsilon^{2}\langle\lambda, \boldsymbol{a}\rangle_{\boldsymbol{\Sigma}} \\
& +\varepsilon^{2} \log \left(\sum_{l=0}^{r}\left(\varepsilon|\lambda|_{\boldsymbol{\Sigma}}\right)^{l}\right)
\end{aligned}
$$

Choose $\lambda$ such that

$$
f(\lambda)=\frac{1}{2} \varepsilon^{4} p|\lambda|_{\Sigma}^{2}+\varepsilon^{2} r|\lambda|_{\Sigma}-\varepsilon^{2}\langle\lambda, \boldsymbol{a}\rangle_{\Sigma}
$$

attains its minimum, which happens when $\lambda$ has the same direction as $\boldsymbol{a}$ since the first two terms only depends on the magnitude of $\lambda$, i.e. we may write $\lambda=\boldsymbol{a}|\lambda|_{\boldsymbol{\Sigma}}|\boldsymbol{a}|_{\boldsymbol{\Sigma}}^{-1}$. Then the function becomes

$$
f(\lambda)=\frac{1}{2} \varepsilon^{4} p|\lambda|_{\Sigma}^{2}+\varepsilon^{2} r|\lambda|_{\Sigma}-\varepsilon^{2}|\boldsymbol{a}|_{\Sigma}|\lambda|_{\Sigma}
$$


which is a quadratic function of $|\lambda|_{\Sigma}$, we thus deduce that it reaches its minimum when

$$
|\lambda|_{\Sigma}=\frac{\left(|\boldsymbol{a}|_{\boldsymbol{\Sigma}}-r\right)^{+}}{\varepsilon^{2} p} .
$$

Therefore, the minimum is attained at

$$
\lambda=\frac{\left(|\boldsymbol{a}|_{\boldsymbol{\Sigma}}-r\right)^{+}}{\varepsilon^{2} p|\boldsymbol{a}|_{\boldsymbol{\Sigma}}} \boldsymbol{a} .
$$

By setting $\lambda$ equal to the above value in Eq. 44, we obtain that

$$
\begin{aligned}
\varepsilon^{2} \log c_{p, r}\left\{\omega: \boldsymbol{B}_{\boldsymbol{t}}(\varepsilon \omega) \in B(\boldsymbol{a}, r)\right\} \leq & -\frac{1}{2 p}\left(\left(|\boldsymbol{a}|_{\boldsymbol{\Sigma}}-r\right)^{+}\right)^{2}+\varepsilon^{2} \log \left(\sum_{l=0}^{r}\left(\frac{\left(|\boldsymbol{a}|_{\boldsymbol{\Sigma}}-r\right)^{+}}{\varepsilon p}\right)^{l}\right) \\
\leq & -\frac{1}{2 p}\left(\left(|\boldsymbol{a}|_{\boldsymbol{\Sigma}}-r\right)^{+}\right)^{2}+\varepsilon^{2} \log (r+1) \\
& +\max _{0 \leq l \leq r} \varepsilon^{2} l \log \left(\frac{\left(|\boldsymbol{a}|_{\boldsymbol{\Sigma}}-r\right)^{+}}{\varepsilon p}\right),
\end{aligned}
$$

which implies that

$$
\begin{aligned}
\limsup _{\varepsilon \rightarrow 0} \varepsilon^{2} \log c_{p, r}\left\{\omega: \boldsymbol{B}_{\boldsymbol{t}}(\varepsilon \omega) \in B(\boldsymbol{a}, r)\right\} & \leq-\frac{1}{2 p}\left(\left(|\boldsymbol{a}|_{\boldsymbol{\Sigma}}-r\right)^{+}\right)^{2} \\
& =-\frac{1}{p} \inf _{\boldsymbol{x} \in B(\boldsymbol{a}, r)} I_{n}(\boldsymbol{x}) .
\end{aligned}
$$

Now for any compact $K \subset\left(\mathbb{R}^{n},|\cdot|_{\boldsymbol{\Sigma}}\right)$ and any $\delta>0$, there exists a finite open cover $\left\{B\left(\boldsymbol{a}_{i}, \delta\right)\right\}_{i \in I}$ in $\left(\mathbb{R}^{n},|\cdot| \boldsymbol{\Sigma}\right)$ of $K$ with $\boldsymbol{a}_{i} \in K$ and $I$ a finite index set. Therefore,

$$
\begin{aligned}
& \limsup _{\varepsilon \rightarrow 0} \varepsilon^{2} \log c_{p, r}\left\{\omega: \boldsymbol{B}_{\boldsymbol{t}}(\varepsilon \omega) \in K\right\} \\
\leq & \limsup _{\varepsilon \rightarrow 0} \varepsilon^{2} \log c_{p, r}\left\{\omega: \boldsymbol{B}_{\boldsymbol{t}}(\varepsilon \omega) \in \bigcup_{i \in I} B\left(\boldsymbol{a}_{i}, \delta\right)\right\} \\
\leq & \limsup _{\varepsilon \rightarrow 0} \varepsilon^{2} \log \left(\sum_{i \in I} c_{p, r}\left\{\omega: \boldsymbol{B}_{\boldsymbol{t}}(\varepsilon \omega) \in B\left(\boldsymbol{a}_{i}, \delta\right)\right\}\right) \\
\leq & \limsup _{\varepsilon \rightarrow 0} \varepsilon^{2} \log |I|+\limsup _{\varepsilon \rightarrow 0} \varepsilon^{2} \log \left(\max _{i \in I} c_{p, r}\left\{\omega: \boldsymbol{B}_{\boldsymbol{t}}(\varepsilon \omega) \in B\left(\boldsymbol{a}_{i}, \delta\right)\right\}\right) \\
= & \max _{i \in I} \limsup _{\varepsilon \rightarrow 0} \varepsilon^{2} \log c_{p, r}\left\{\omega: \boldsymbol{B}_{\boldsymbol{t}}(\varepsilon \omega) \in B\left(\boldsymbol{a}_{i}, \delta\right)\right\} \\
\leq & \max _{i \in I}-\frac{1}{p} \inf _{\boldsymbol{x} \in B\left(\boldsymbol{a}_{i}, \delta\right)} I_{n}(\boldsymbol{x}) \\
= & -\frac{1}{p} \min _{i \in I} \inf _{\boldsymbol{x} \in B\left(\boldsymbol{a}_{i}, \delta\right)} I_{n}(\boldsymbol{x}) \\
\leq & -\frac{1}{p} \inf _{\boldsymbol{x} \in B(K, \delta)} I_{n}(\boldsymbol{x})
\end{aligned}
$$

where $B(K, \delta)=\left\{\boldsymbol{x} \in \mathbb{R}^{n}: \inf _{y \in K}|\boldsymbol{x}-\boldsymbol{y}|_{\boldsymbol{\Sigma}}<\delta\right\}$. Let $\delta \rightarrow 0$, then the upper bound is established for all compact sets. 
Now for any $F \subset \mathbb{R}^{n}$ closed under Euclidean metric, as all norms on $\mathbb{R}^{n}$ are equivalent, $F$ is also closed in $\left(\mathbb{R}^{n},|\cdot| \boldsymbol{\Sigma}\right)$. For $\rho>0$, let $H_{\rho}=\left\{\boldsymbol{x}=\left(x_{1}, \cdots, x_{n}\right):\left|x_{i}\right| \leq \rho, \forall 1 \leq i \leq n\right\}$ be a hypercube in $\mathbb{R}^{n}$. Then by sub-additivity property,

$$
\begin{aligned}
c_{p, r}\left\{\omega: \boldsymbol{B}_{\boldsymbol{t}}(\varepsilon \omega) \in F\right\} & \leq c_{p, r}\left\{\omega: \boldsymbol{B}_{\boldsymbol{t}}(\varepsilon \omega) \in F \cap H_{\rho}\right\}+c_{p, r}\left\{\omega: \boldsymbol{B}_{\boldsymbol{t}}(\varepsilon \omega) \in H_{\rho}^{C}\right\} \\
& \leq c_{p, r}\left\{\omega: \boldsymbol{B}_{\boldsymbol{t}}(\varepsilon \omega) \in F \cap H_{\rho}\right\}+\sum_{i=1}^{n} c_{p, r}\left\{\omega:\left|B_{t_{i}}(\varepsilon \omega)\right|>\rho\right\} .
\end{aligned}
$$

Therefore, by the result for compact sets and Eq. 42, as well as Lemma 1.2.15 on page 7 in [7], we have that

$$
\begin{aligned}
& \limsup _{\varepsilon \rightarrow 0} \varepsilon^{2} \log c_{p, r}\left\{\omega: \boldsymbol{B}_{\boldsymbol{t}}(\varepsilon \omega) \in F\right\} \\
\leq & \max \left\{\limsup _{\varepsilon \rightarrow 0} \varepsilon^{2} \log c_{p, r}\left\{\omega: \boldsymbol{B}_{\boldsymbol{t}}(\varepsilon \omega) \in F \cap H_{\rho}\right\},\right. \\
& \left.\limsup _{\varepsilon \rightarrow 0} \varepsilon^{2} \log \left(\sum_{i=1}^{n} c_{p, r}\left\{\omega:\left|B_{t_{i}}(\varepsilon \omega)\right|>\rho\right\}\right)\right\} \\
\leq & \max \left\{\limsup _{\varepsilon \rightarrow 0} \varepsilon^{2} \log c_{p, r}\left\{\omega: \boldsymbol{B}_{\boldsymbol{t}}(\varepsilon \omega) \in F \cap H_{\rho}\right\},\right. \\
& \left.\limsup _{\varepsilon \rightarrow 0} \varepsilon^{2} \log \left(c_{p, r}\left\{\omega:\left|B_{t_{i}}(\varepsilon \omega)\right|>\rho\right\}\right)\right\} \\
\leq & \max \left\{-\frac{1}{p} \inf _{\boldsymbol{x} \in F \cap H_{\rho}} I_{n}(\boldsymbol{x}),-\frac{1}{p} \inf _{x>\rho} I_{1}(x)\right\}
\end{aligned}
$$

for all $\rho>0$. The proof is complete by letting $\rho \rightarrow \infty$.

Now we may conclude our proof of the quasi-sure large deviation principle, the second part of Theorem 2 .

Proof of Theorem 2, (2) Since $F_{m}: \mathbb{R}^{2^{m}+1} \rightarrow \boldsymbol{W}$ defined in Eq. 40 is continuous and $F_{m} \circ T^{\varepsilon}=X^{\varepsilon,(m)}$, by the contraction principle (Theorem 4), the family $\left\{X^{\varepsilon,(m)}\right\}$ satisfies the $c_{p, r}$-LDP with the good rate function

$$
J_{m}(\omega)=\inf _{\boldsymbol{x}: F_{m}(\boldsymbol{x})=\omega} I_{2^{m}+1}(\boldsymbol{x}), \quad \omega \in \boldsymbol{W},
$$

where we define $\inf \emptyset=\infty$. When $p=1$ and $r=0$, the capacity $c_{p, r}$ coincides with Wiener measure $\mathbb{P}$, and we would expect that the classical LDP for fBM defined on the classical Wiener space holds.

Now define $\hat{F}_{m}: \boldsymbol{W} \rightarrow \boldsymbol{W}$ by

$\hat{F}_{m}(\omega)=\omega\left(\frac{k-1}{2^{m}}\right)+2^{m}\left(t-\frac{k-1}{2^{m}}\right)\left(\omega\left(\frac{k}{2^{m}}\right)-\omega\left(\frac{k-1}{2^{m}}\right)\right), \quad t \in\left[\frac{k-1}{2^{m}}, \frac{k}{2^{m}}\right]$,

which is a continuous mapping with respect to the uniform convergence topology. Then by the classical contraction principle for measures (see Theorem 4.2.1 on page 126 in [7]), the family $\left\{\mathbb{Q}_{\varepsilon} \circ \hat{F}_{m}^{-1}\right\}$ on $(\boldsymbol{W}, \mathscr{B}(\boldsymbol{W}))$ satisfies the LDP with the good rate function

$$
\hat{J}_{m}(\omega)=\inf \left\{I(x): x \in \boldsymbol{W}, \hat{F}_{m}(x)=\omega\right\}, \quad \forall \omega \in \boldsymbol{W},
$$


where inf $\emptyset=\infty$. By Eq. 3, for each $A \in \mathscr{B}(\boldsymbol{W}), \hat{F}_{m}^{-1}(A) \in \mathscr{B}(\boldsymbol{W})$, and

$$
\begin{aligned}
\mathbb{Q}_{\varepsilon} \circ \hat{F}_{m}^{-1}(A) & =\mathbb{Q}_{\varepsilon}\left(\hat{F}_{m}^{-1}(A)\right) \\
& =\mathbb{P}\left\{\omega \in \boldsymbol{W}: \varepsilon B(\omega) \in \hat{F}_{m}^{-1}(A)\right\} \\
& =\mathbb{P}\left\{\omega \in \boldsymbol{W}: \hat{F}_{m}(\varepsilon B(\omega)) \in A\right\} .
\end{aligned}
$$

Since $\hat{F}_{m}(\varepsilon B)=X^{\varepsilon,(m)} \mathbb{P}$-a.s. on $\boldsymbol{W}$, we obtain that

$$
\mathbb{Q}_{\varepsilon} \circ \hat{F}_{m}^{-1}(A)=\mathbb{P}\left\{\omega \in \boldsymbol{W}: X^{\varepsilon,(m)}(\omega) \in A\right\} .
$$

Therefore, by the uniqueness of rate functions (see Lemma 4.1.4, Section 4.1.1 in [7]), $J_{m}$ coincides with $\hat{J}_{m}$.

As shown in Theorem 10, $\left\{X^{\varepsilon,(m)}: \varepsilon>0\right\}$ are exponentially good approximations of $\left\{X^{\varepsilon}: \varepsilon>0\right\}$, so it suffices to verify that the function $I$ defined above coincides with the function $J$ given in Eq. 9 and satisfies all conditions in Proposition 6. Let us first check if $I$ satisfies all conditions. We observe that $I$ given in Eq. 4 is a good rate function by definition.

For any closed $C \subset \boldsymbol{W}$, denote $\eta_{m}=\inf _{\omega \in C} \hat{J}_{m}(\omega)$, where $\hat{J}_{m}=J_{m}$ is defined as in Eq. 46. By definition, $\eta_{m}=\inf _{\omega \in \hat{F}_{m}^{-1}(C)} I(\omega)$. Suppose that $\liminf _{m \rightarrow \infty} \eta_{m}=\eta<\infty$, then as $I$ is a good rate function and lower semi-continuous functions attain their minimums on compact sets, we conclude that $I$ attains its minimum on the closed subset $\hat{F}_{m}^{-1}(C) \in \boldsymbol{W}$. Therefore, for each $m$, there exists some $\omega_{m} \in \boldsymbol{W}$ such that $\omega_{m} \in \hat{F}_{m}^{-1}(C)$ and $\eta_{m}=$ $\inf _{\omega \in \hat{F}_{m}^{-1}(C)} I(\omega)=I\left(\omega_{m}\right)$.

We notice that for all $\omega \in \boldsymbol{W}, \hat{F}_{m}(\omega) \rightarrow \omega$ in $(W,\|\cdot\|)$ as $m \rightarrow \infty$. Since $\hat{F}_{m}\left(\omega_{m}\right) \in C$ for all $m$, for each $\delta>0, \omega_{m} \in C_{\delta}$ for large $m$, where $C_{\delta}=\{\omega:\|\omega-C\| \leq \delta\}$. It follows that

$$
\inf _{\omega \in C_{\delta}} I(\omega) \leq I\left(\omega_{m}\right)=\eta_{m}=\inf _{\omega \in C} \hat{J}_{m}(\omega)
$$

for $m$ sufficiently large, and hence by taking limit infimum on both sides, we deduce that

$$
\inf _{\omega \in C_{\delta}} I(\omega) \leq \liminf _{m \rightarrow \infty} \inf _{\omega \in C} \hat{J}_{m}(\omega) .
$$

According to Lemma 4.1.6 (a), Section 4.1.1 in [7],

$$
\inf _{\omega \in C} I(\omega) \leq \liminf _{m \rightarrow \infty} \inf _{\omega \in C} \hat{J}_{m}(\omega)
$$

when letting $\delta \rightarrow 0$, and hence the condition (10) is fulfilled. The case when $\liminf _{m \rightarrow \infty} \eta_{m}=\infty$ is trivial, so we have verified all conditions in Proposition 6.

Next, we prove that $I$ coincides with the function $J$ defined as in Eq. 9 by

$$
J(\omega)=\sup _{\lambda>0} \liminf _{m \rightarrow \infty} \inf _{x \in B(\omega, \lambda)} \hat{J}_{m}(x) .
$$

For any $\omega \in \boldsymbol{W}$, set $C=\overline{B(\omega, \lambda)}$ in Eq. 47. It holds that

$$
\inf _{x \in \overline{B(\omega, \lambda)}} I(x) \leq \liminf _{m \rightarrow \infty} \inf _{x \in \overline{B(\omega, \lambda)}} \hat{J}_{m}(x) \leq \liminf _{m \rightarrow \infty} \inf _{x \in B(\omega, \lambda)} \hat{J}_{m}(x) \leq J(\omega) .
$$

By letting $\lambda \rightarrow 0$ and applying Lemma 4.1.6 (a), Section 4.1.1 in [7], we conclude that $I(\omega) \leq J(\omega)$ for all $\omega$. For the reverse part, denote $\hat{\omega}_{m}=\hat{F}_{m}(\omega)$ for $\omega \in \boldsymbol{W}$, and as shown above, $\hat{\omega}_{m} \rightarrow \omega$ as $m \rightarrow \infty$. Therefore, for any $\lambda>0$, there exists some $M>0$ such that for all $m \geq M$,

$$
\inf _{x \in B(\omega, \lambda)} \hat{J}_{m}(x) \leq \hat{J}_{m}\left(\hat{\omega}_{m}\right)
$$


By the definition of $\hat{J}_{m}, \hat{J}_{m}\left(\hat{\omega}_{m}\right) \leq I(\omega)$. By taking limit infimum over $m$ first, then supremum over $\lambda$, we obtain that

$$
J(\omega)=\sup _{\lambda>0} \liminf _{m \rightarrow \infty} \inf _{x \in B(\omega, \lambda)} \hat{J}_{m}(x) \leq I(\omega),
$$

and hence $I=J$.

Open Access This article is licensed under a Creative Commons Attribution 4.0 International License, which permits use, sharing, adaptation, distribution and reproduction in any medium or format, as long as you give appropriate credit to the original author(s) and the source, provide a link to the Creative Commons licence, and indicate if changes were made. The images or other third party material in this article are included in the article's Creative Commons licence, unless indicated otherwise in a credit line to the material. If material is not included in the article's Creative Commons licence and your intended use is not permitted by statutory regulation or exceeds the permitted use, you will need to obtain permission directly from the copyright holder. To view a copy of this licence, visit http://creativecommonshorg/licenses/by/4.0/.

\section{References}

1. Airault, H.: Differential calculus on finite codimensional submanifolds of the Wiener space-The divergence operator. J. Funct. Anal. 100(2), 291-316 (1991)

2. Albeverio, S., Fukushima, M., Hansen, W., Ma, Z., Röckner, M.: An invariance result for capacities on Wiener space. J. Funct. Anal. 106(1), 35-49 (1992)

3. Biagini, F., Hu, Y., Øksendal, B., Zhang, T. Stochastic calculus for fractional Brownian motion and applications. Springer Science \& Business Media (2008)

4. Boedihardjo, H., Geng, X., Qian, Z.: Quasi-sure existence of gaussian rough paths and large deviation principles for capacities. Osaka J. Math. 53(4), 941-970 (2016)

5. Bogachev, V.I.: Gaussian measures. Surv. American Mathematical Society, Providence (1998)

6. Decreusefond, L., Üstünel, A.S.: Stochastic analysis of the fractional Brownian motion. Potential Anal. 10(2), 177-214 (1999)

7. Dembo, A., Zeitouni, O. Large Deviations Techniques and Applications, 2nd edn., vol. 38. Springer, London (1998)

8. Denis, L.: Analyse quasi-sûre de l'approximation d'Euler et du flot d'une EDS. Comptes rendus de 1'Académie des sciences. Série 1 Mathématique 315(5), 599-602 (1992)

9. Deuschel, J.-D., Stroock, D.W.: Large Deviations, vol. 342. American Mathematical Soc. (2001)

10. Donsker, M.D.: Asymptotic evaluation of certain Wiener integrals for large time. In: Proceedings of International Conference on Function Space, Oxford (1974)

11. Fang, S.: Le calcul différentiel quasi-sur et son application à l'estimation du noyau de la chaleur. Comptes rendus de l'Académie des sciences. Série 1 Mathématique 311(6), 369-372 (1990)

12. Friz, P., Victoir, N.: Large deviation principle for enhanced Gaussian processes. Annales de l'Institut Henri Poincare / Probabilites et statistiques 43(6), 775-785 (2007)

13. Friz, P.K., Hairer, M.: A Course on Rough Paths: with an Introduction to Regularity Structures, UTX. Springer, Cham (2014)

14. Friz, P.K., Victoir, N.B.: Multidimensional Stochastic Processes as Rough Paths: Theory and Applications. CSAM. Cambridge University Press, Cambridge (2010)

15. Fukushima, M., Kaneko, H.: On $(r, p)$-capacities for general Markovian semigroups. In: Albeverio, S., Pitman (eds.) Infinite Dimensional Analysis and Stochastic Processes (1985)

16. Fukushima, M., Oshima, Y., Takeda, M.: Dirichlet Forms and Symmetric Markov Processes. Ebook central. De Gruyter, New York, 2nd rev. and ext. edition (2010)

17. Gao, F., Liu, J.: Large deviations for small perturbations of sdes with non-Markovian coefficients and their applications. Stoch. Dyn. 6(04), 487-520 (2006)

18. Gao, F., Ren, J.: Large deviations for stochastic flows and their applications. Sci. China Series A: Math. 44(8), 1016-1033 (2001)

19. Huang, Z., Ren, J.: Quasi sure stochastic flows. Stochastics and Stochastic Reports 33(3-4), 149-157 (1990)

20. Ikeda, N., Watanabe, S.: Stochastic Differential Equations and Diffusion Processes, vol. 24. Elsevier (2014)

21. Inahama, Y.: Quasi-sure existence of Brownian rough paths and a construction of Brownian pants. Infinite Dimensional Analysis, Quantum Probability and Related Topics 9(04), 513-528 (2006) 
22. Inahama, Y.: Laplace approximation for rough differential equation driven by fractional Brownian motion. Ann. Probab. 41(1), 170 (2013)

23. Inahama, Y.: Large deviation principle for certain spatially lifted Gaussian rough path. Tohoku. Math. J. (2) 67(3), 433-463 (2015a)

24. Inahama, Y.: Large deviation principle of Freidlin-Wentzell type for pinned diffusion processes. Trans. Am. Math. Soc. 367(11), 8107-8137 (2015b)

25. Kaneko, H.: On $(r, p)$-capacities for Markov processes. Osaka J. Math. 23(2), 325-336 (1986)

26. Kazumi, T.: Refinement in terms of capacities of certain limit theorems on an abstract Wiener space. J. Math. Kyoto Univ. 32(1), 1-29 (1992)

27. Kazumi, T., Shigekawa, I.: Measures of finite $(r, p)$-energy and potentials on a separable metric space. In: Séminaire de Probabilités XXVI, pp. 415-444. Springer (1992)

28. Ledoux, M., Qian, Z., Zhang, T.: Large deviations and support theorem for diffusion processes via rough paths. Stoch. Process. Appl. 102(2), 265-283 (2002)

29. Li, J., Qian, Z.: Fine properties of fractional Brownian motions on Wiener space. J. Math. Anal. Appl. 473(1), 141-173 (2019)

30. Liu, J., Ren, J.: A functional modulus of continuity for Brownian motion. Bull. des Sciences Mathématiques 131(1), 60-71 (2007)

31. Lyons, T., Qian, Z.: System Control and Rough Paths. Oxford University Press (2002)

32. Lyons, T.J., Caruana, M., Lévy, T.: Differential Equations Driven by Rough Paths: École d'été de probabilités de Saint-Flour XXXIV-2004. L.N.M. Springer, New York (2007)

33. Malliavin, P.: $C^{k}$-Hypoellipticity with Degeneracy, Stochastic Analysis. In: Friedman, A., Pinsky, M. (eds.), pp. 199-214, 321-340 (1978)

34. Malliavin, P.: Stochastic calculus of variations and hypoelliptic operators. In: Proc. Internat. Symposium on Stochastic Differential Equations. Kyoto Univ., p. 1976. Wiley, Kyoto (1978)

35. Malliavin, P.: Stochastic Analysis Comprehensive Studies in Mathematics. Springer, London (1997)

36. Malliavin, P., Nualart, D.: Quasi sure analysis and Stratonovich anticipative stochastic differential equations. Probab. Theory Relat. Fields 96(1), 45-55 (1993)

37. Malliavin, P., Nualart, D.: Quasi sure analysis of stochastic flows and Banach space valued smooth functionals on the Wiener space. J. Funct. Anal. 112(2), 287-317 (1993)

38. Mandelbrot, B.B., Van Ness, J.W.: Fractional Brownian motions, fractional noises and applications. SIAM Rev. 10(4), 422-437 (1968)

39. Millet, A., Sanz-Solé, M.: Large deviations for rough paths of the fractional Brownian motion. Annales de l'Institut Henri Poincare / Probabilites et statistiques 42(2), 245-271 (2006)

40. Mishura, Y.: Stochastic Calculus for Fractional Brownian Motion and Related Processes L.N.M. Springer, Berlin (2008)

41. Nualart, D. The Malliavin Calculus and Related Topics. Probability and its Applications, 2nd edn. Springer, Berlin (2006)

42. Ren, J.: Analysis quasi sûre des équation différentielles stochastiques. Bulletin des Sciences Mathématiques 114(2), 187-213 (1990)

43. Ren, J.: Analyse quasi sûre des martingales régulières. Comptes rendus de l'Académie des sciences. Série 1 Mathématique 317(3), 299-302 (1993)

44. Varadhan, S.R.S.: Large deviations and applications. Conference Board of the Mathematical Sciences. CBMS-NSF regional conference series in applied mathematics Society for Industrial and Applied Mathematics. Philadelphia (1984)

45. Yoshida, N.: A large deviation principle for $(r, p)$-capacities on the Wiener space. Probab. Theory Relat. Fields 94(4), 473-488 (1993)

Publisher's Note Springer Nature remains neutral with regard to jurisdictional claims in published maps and institutional affiliations. 